OPEN ACCESS

Edited by:

Nicolas Durand,

Université Pierre et Marie Curie,

France

Reviewed by:

Andrew Dacks,

West Virginia University, United States

Julian Chen,

Institute of Plant Protection (CAS),

China

*Correspondence:

Ewald Grosse-Wilde

grosse-wilde@ice.mpg.de

Patrizia Falabella

patrizia.falabella@unibas.it

tThese authors have contributed equally to this work.

Specialty section: This article was submitted to Invertebrate Physiology, a section of the journal

Frontiers in Physiology

Received: 19 December 2017

Accepted: 04 June 2018

Published: 25 June 2018

Citation

Bruno D, Grossi G, Salvia R, Scala A,

Farina D, Grimaldi A, Zhou J-J, Bufo SA, Vogel H, Grosse-Wilde E, Hansson BS and Falabella P (2018) Sensilla Morphology and Complex

Expression Pattern of Odorant Binding Proteins in the Vetch Aphid Megoura viciae (Hemiptera: Aphididae). Front. Physiol. 9:777. doi: 10.3389/fphys.2018.00777

\section{Sensilla Morphology and Complex Expression Pattern of Odorant Binding Proteins in the Vetch Aphid Megoura viciae (Hemiptera: Aphididae)}

\author{
Daniele Bruno ${ }^{1 \dagger}$, Gerarda Grossi ${ }^{2 \dagger}$, Rosanna Salvia ${ }^{2 \dagger}$, Andrea Scala ${ }^{2+}$, Donatella Farina ${ }^{2}$, \\ Annalisa Grimaldi ${ }^{1}$, Jing-Jiang Zhou ${ }^{3}$, Sabino A. Bufo ${ }^{2}$, Heiko Vogel $^{4}$, \\ Ewald Grosse-Wilde ${ }^{5 *}$, Bill S. Hansson ${ }^{5}$ and Patrizia Falabella ${ }^{2 *}$
}

${ }^{1}$ Department of Biotechnology and Life Sciences, University of Insubria, Varese, Italy, ${ }^{2}$ Department of Sciences, University of Basilicata, Potenza, Italy, ${ }^{3}$ Department of Biological Chemistry and Crop Protection, Rothamsted Research, Harpenden, United Kingdom, ${ }^{4}$ Department of Entomology, Max Planck Institute for Chemical Ecology, Jena, Germany, ${ }^{5}$ Department of Evolutionary Neuroethology, Max Planck Institute for Chemical Ecology, Jena, Germany

Chemoreception in insects is mediated by several components interacting at different levels and including odorant-binding proteins (OBPs). Although recent studies demonstrate that the function of OBPs cannot be restricted to an exclusively olfactory role, and that OBPs have been found also in organs generally not related to chemoreception, their feature of binding molecules remains undisputed. Studying the vetch aphid Megoura viciae (Buckton), we used a transcriptomic approach to identify ten OBPs in the antennae and we examined the ultrastructural morphology of sensilla and their distribution on the antennae, legs, mouthparts and cauda of wingless and winged adults by scanning electron microscopy (SEM). Three types of sensilla, trichoid, coeloconic and placoid, differently localized and distributed on antennae, mouthparts, legs and cauda, were described. The expression analysis of the ten OBPs was performed by RT-qPCR in the antennae and other body parts of the wingless adults and at different developmental stages and morphs. Five of the ten OBPs (MvicOBP1, MvicOBP3, MvicOBP6, MvicOBP7, and MvicOBP8), whose antibodies were already available, were selected for experiments of whole-mount immunolocalization on antennae, mouthparts, cornicles and cauda of adult aphids. Most of the ten OBPs were more expressed in antennae than in other body parts; MvicOBP1, MvicOBP3, MvicOBP6, MvicOBP7 were also immunolocalized in the sensilla on the antennae, suggesting a possible involvement of these proteins in chemoreception. MvicOBP6, MvicOBP7, MvicOBP8, MvicOBP9 were highly expressed in the heads and three of them (MvicOBP6, MvicOBP7, MvicOBP8) were immunolocalized in the sensilla on the mouthparts, supporting the hypothesis that also mouthparts may be involved in chemoreception. MvicOBP2, MvicOBP3, MvicOBP5, MvicOBP8 were highly expressed in the cornicles-cauda and two of them (MvicOBP3, MvicOBP8) were immunolocalized in cornicles and in cauda, suggesting a possible new function not related to chemoreception. Moreover, the response of $M$. viciae to different components 
of the alarm pheromone was assessed by behavioral assays on wingless adult morph; $(-)-\alpha$-pinene and $(+)$-limonene were found to be the components mainly eliciting an alarm response. Taken together, our results represent a road map for subsequent indepth analyses of the OBPs involved in several physiological functions in M. viciae, including chemoreception.

Keywords: vetch aphid, chemoreception, odorant-binding proteins, RT-qPCR, immunolocalization, behavioral assays

\section{INTRODUCTION}

Chemical perception in insects is known to be mediated by molecules belonging to the classes of olfactory, gustatory and ionotropic receptors and to the classes of soluble olfactory proteins, odorant-binding proteins (OBPs) and chemosensory proteins (CSPs); however, what these proteins do and how they interact is still not completely clear (Fan et al., 2010; Leal, 2013; Pelosi et al., 2017). In particular, OBPs have long been thought to act exclusively as carriers of chemicals that, once solubilized, were transported to the olfactory receptors (Pelosi et al., 2006; Brito et al., 2016). The generally hydrophobic odorants need to reach the specific receptors bound to the plasma membrane of sensory neuron dendrites, overcoming the hydrophilic barrier that is the sensillar lymph (Pelosi, 1996; Jeong et al., 2013). Several studies performed in vivo indicate that OBPs play a key role in chemoreception. RNAi was used to reduce the expression of OBPs in Anopheles gambiae and Culex quinquefasciatus (Biessmann et al., 2010; Pelletier et al., 2010), in Drosophila melanogaster (Swarup et al., 2011) and in Acyrthosiphon pisum (Zhang et al., 2017). Results demonstrated that OBPs play a specific role in olfactory perception, suggesting there is a direct correlation between the expression level of OBPs and the ability of insects to perceive odors. Previous studies found that Drosophila OBP76a (LUSH) mutants, played an essential role in binding and mediating the recognition of the sex pheromone 11-cis-vaccenyl acetate (c-VA) (Xu et al., 2005; $\mathrm{Ha}$ and Smith, 2006; Laughlin et al., 2008). These preliminary results should be partially reconsidered in light of more recent research demonstrating that, at sufficiently high concentrations, c-VA is able to activate neuronal stimuli without LUSH (GomezDiaz et al., 2013; Li et al., 2014). However, LUSH is still considered a protein that can increase the sensitivity of the c-VA receptor, also protecting pheromone molecules from degradation by ODEs (Gomez-Diaz et al., 2013). Moreover, the capability of LUSH to bind c-VA has been further demonstrated by in vitro experiments (Kruse et al., 2003).

It has been demonstrated that deleting OBP28a in Drosophila melanogaster basiconic sensilla did not reduce the insect's ability to respond to olfactory stimuli (Larter et al., 2016), suggesting that OBP28a is not required for odorant transport. Larter and colleagues hypothesize a novel role for OBP, namely, that it modulates odor perception by mitigating the effect of rapid changes in the level of environmental odors. In their model, odorants are transported from the sensillum pore to the sensory neuron through hydrophobic tunnels called pore tubules (Steinbrecht, 1997). However, since in Drosophila melanogaster only basiconic sensilla contain pore tubules (Shanbhag et al., 2000), the authors do not exclude that OBP28a expressed in other sensilla type may play different roles including the classical function of odorants carrier (Larter et al., 2016).

Alternatively, different studies suggest that a sensible reduction in olfactory function is related to the reduced levels of certain OBPs (Xu et al., 2005; Biessmann et al., 2010; Pelletier et al., 2010; Swarup et al., 2011). Within the processes relying on chemoreception, it has been proposed that OBPs also play a role in removing chemicals, both those bound to the ORs and those located in the sensory lymph, in order to speed up nervous stimulus termination (Vogt and Riddiford, 1981; Ziegelberger, 1995). That the role of OBPs is related to their binding task is apparent from their multifunctional features, which are not confined to chemical perception (Smartt and Erickson, 2009; Sun Y.L. et al., 2012; Ishida et al., 2013; Pelosi et al., 2017). Indeed, OBPs are expressed in organs that are not connected to chemoreception. In some cases, the same OBP is expressed in chemoreceptive and non-chemoreceptive tissues, suggesting that one type may have multiple roles (Calvello et al., 2003; Li et al., 2008; Sirot et al., 2008; Vogel et al., 2010; Dani et al., 2011; Iovinella et al., 2011; Sun Y.L. et al., 2012; De Biasio et al., 2015). For example, since the same OBPs are expressed in antennae and reproductive organs (Sun Y.L. et al., 2012; Ban et al., 2013), or in antennae and in pheromone glands (Jacquin-Joly et al., 2001; Strandh et al., 2009; Gu et al., 2013; Zhang et al., 2013, 2015; Xia et al., 2015), they may both mediate the recognition of and assisting with the release of the same chemical message. In both cases, the role of OBPs is to solubilize hydrophobic pheromones, binding them in a hydrophilic environment where OBPs are present in high concentration (Nagnan-Le Meillour et al., 2000; Jacquin-Joly et al., 2001; Pelosi et al., 2017).

The different functions imputed to OBPs are in any case linked to the ability of these proteins to bind small hydrophobic molecules, signals of different types originating from different sources. However, the expression of soluble olfactory proteins in chemosensory structures (mainly antennae and mouthparts) indicates that they play a role in chemoreception (Pelosi et al., 2017).

Chemoreception is just one of the roles that OBPs play in aphids (Hemiptera: Aphididae), a group of insects that includes major crop pest in the world. Aphids cause damage directly and indirectly, by feeding and transmitting plant viruses (Nault, 1997; Hogenhout et al., 2008; Webster, 2012). Aphids use their olfactory system and semiochemicals, such as plant volatiles and pheromones, for many purposes: to locate their host plants, select a partner, and escape from danger 
(van Emden and Harrington, 2007). In aphids, as in other insects, OBPs have the capability to transport semiochemicals across the sensillar lymph toward the ORs located on the sensory neuron membrane (Qiao et al., 2009; Vandermoten et al., 2011; Sun Y.F. et al., 2012). Even if the mode of action of OBPs is not completely understood, the chemical message is known to be transduced into a neuronal impulse that starts at the dendrite of the olfactory sensory neuron (Leal, 2013); next, the signal reaches the antennal lobe in the brain, where it is processed and leads to a behavioral response (Distler and Boeckh, 1996; Fan et al., 2010).

In the present work, we adopted a multidisciplinary approach to study chemoreception in the vetch aphid Megoura viciae (Buckton), which feeds exclusively on members of Leguminosae (Nuessly et al., 2004).

After constructing and analyzing the $M$. viciae antennal transcriptome, we identified the OBPs expressed in antennae and determined their expression using the reads per kilobase per million mapped reads (RPKM) method. The expression profile of all the identified OBPs at different developmental stages and in different body parts was also analyzed by RT-qPCR. Moreover, whole mount immunolocalization of five identified OBPs was performed using available antibodies. In addition, scanning electron microscopy (SEM) was carried out on antennae, legs, mouthparts and cauda of both wingless and winged adult morphs to scrutinize the morphology of sensilla expressing the analyzed OBPs at the ultrastructural level. Furthermore, we performed a behavioral assay using the different components of $M$. viciae's alarm pheromone.

Although our study focuses on the typical chemoreceptive organ, the antennae, and investigates how the expression of OBPs supports the putative role in olfactory and gustatory perception, our results suggest that these soluble proteins play other roles in addition to chemoreception.

\section{MATERIALS AND METHODS}

\section{Insect Rearing and Sample Collection}

Megoura viciae was reared on potted broad bean plants (Vicia faba L.) at $24 \pm 1^{\circ} \mathrm{C}, 75 \% \pm 5 \% \mathrm{RH}$ and $16 \mathrm{~h}$ light $-8 \mathrm{~h}$ dark photoperiod. Aphid cultures were started with insects originally collected from broad bean plants in southern Italy near Salerno $\left(40^{\circ} 37^{\prime} \mathrm{N} ; 15^{\circ} 3^{\prime} \mathrm{E}\right)$. In order to synchronize aphid nymphal instars, parthenogenetic females were placed on potted broad bean plants; newborn aphids were separated as soon as they appeared, and adults were removed from plants. Newborn aphids were maintained on plants for 6 days and collected at different developmental stages, from first nymphal instar to adults, both wingless (apterous) and winged (alatae) morphs. Samples were frozen using liquid nitrogen and stored at $-80^{\circ} \mathrm{C}$ until the RNA extraction used for RT-qPCR experiments. Antennae, de-antennaed heads, legs, cornicles, cauda and remaining body parts of wingless adult aphids were dissected under the microscope, fixed and prepared for SEM, immunolocalization experiments, or homogenized in TRI Reagent (Sigma, St. Louis, $\mathrm{MO}$, United States) and stored at $-80^{\circ} \mathrm{C}$ until the RNA extraction used for RT-qPCR experiments. Wingless adults were used in behavior experiments. Some specimens deriving from the described original strain were sent to the Department of Biological Chemistry and Crop Protection, Rothamsted Research, Harpenden, United Kingdom, where aphids were reared in the same conditions described above $\left(24 \pm 1^{\circ} \mathrm{C}, 75 \% \pm 5 \% \mathrm{RH}\right.$ and $16 \mathrm{~h}$ light $-8 \mathrm{~h}$ dark photoperiod). Antennae cut from wingless adults were used for RNA extraction and sequencing at the Beijing Genomics Institute (BGI).

\section{Scanning Electron Microscopy (SEM)}

Adult aphids ( 6 in the wingless morph and 2 in the winged morph) were prepared as described by Sun et al. (2013). Briefly, they were fixed in $70 \%$ ethanol for $2 \mathrm{~h}$ and cleaned in an ultrasonic bath for $1 \mathrm{~min}$ in the same solution. Finally, samples were dehydrated in $100 \%$ ethanol for $30 \mathrm{~min}$, airdried, coated in gold by K250 sputter coater (Emitech, Ashford, Kent, United Kingdom) and examined with SEM-FEG XL-30 microscope (Philips, Eindhoven, The Netherlands).

\section{Total RNA Extraction and cDNA Synthesis}

Total RNA, collected from 800 antennae, 80 de-antennaed heads, 500 legs, 500 cornicles-cauda and 40 remaining body parts of wingless adult aphids and from 30 aphids of each different nymphal instar (I, II, III, IV) and each adult morph, was extracted using TRI Reagent (Sigma, St. Louis, MO, United States), following the manufacturer's instructions. The concentration of total RNA was measured spectrophotometrically at $260 \mathrm{~nm}$, using a NanoDrop ND-1000 instrument (Nanodrop Technologies, Wilmington, DE, United States). The purity of RNA was estimated at absorbance ratios OD260/280 and OD260/230, and the integrity was verified on $0.8 \%$ agarose gel electrophoresis. In order to efficiently remove genomic DNA contamination, the samples were treated with $1 \mathrm{U}$ of DNase I (Deoxyribonuclease I, Amplification Grade, Invitrogen-Life Technologies, Carlsbad, CA, United States) per microgram of RNA for $15 \mathrm{~min}$ at room temperature, following the manufacturer's guidelines. cDNA was synthesized using the SuperScript III First-Strand Synthesis System for RT-qPCR (Invitrogen-Life Technologies), according to the manufacturer's protocol, using $5 \mu \mathrm{g}$ of total RNA per sample. The cDNA synthesis reaction was diluted with nucleasefree water to a final volume of $100 \mu \mathrm{l}$ and immediately used for RT-qPCR studies or stored at $-20^{\circ} \mathrm{C}$.

\section{RNA-Seq Data Generation and de novo Transcriptome Assembly}

Antennal transcriptome sequencing was performed with poly(A)enriched mRNA fragmented to an average of 150 nucleotides. Sequencing was carried out by the BGI using paired-end sequencing on an Illumina HiSeq2000 sequencer.

After transformation to raw data, low quality (reads with unknown sequences ' $N$ ') adaptor sequences were removed; reads with certain lengths of overlap were combined to form longer fragments, called contigs. These contigs were subjected to further processing of sequence clustering to form longer sequences without N. Such sequences were defined as unigenes. 
Reads were trimmed of adapters using Cutadapt (Martin, 2011), and of bad quality regions using Sickle (Joshi and Fass, 2011). Subsequently, reads were assembled using Trinity 2.2 with default parameters (Grabherr et al., 2011).

\section{Annotation of OBP Coding Transcripts}

The base of the annotation was a hand-curated database of OBP proteins which, among others, contained known aphid candidate protein sequences. The assembled sequences were compared with the references dataset using blastx. All sequences that generated a hit were further scrutinized by blastx comparison against the NCBI non-redundant database (nr), removing any sequences with evidence for an identity that differs from OBP. Finally, the remaining candidates were translated and aligned with the references using MAFFT (Katoh and Standley, 2013), removing candidates that did not align well with known OBP protein sequences. During this step, candidates were also scrutinized for the presence of the conserved OBP cysteine-pattern.

\section{Quantitative Real Time PCR (RT-qPCR)}

RT-qPCR experiments were carried out in a 7500 Fast Real-Time PCR System (Applied Biosystems- Life Technologies, Carlsbad, CA, United States), on cDNA samples prepared from 5 different nymphal instars, including winged and wingless morphs, and from different body parts (antennae, de-antennaed heads, legs, cornicles and cauda and remaining body parts) of wingless adults. Ribosomal protein S9 (RPS9) and ribosomal protein L32 (RPL32), whose use was validated in a previous work (Cristiano et al., 2016), were chosen as reference genes for the normalization of data obtained from aphids of different nymphal instars and aphids' different body parts RT-qPCR, following the guidelines reported in minimum information required for publication of quantitative real-time PCR experiments (MIQE) (Bustin et al., 2009) and minimum information necessary for quantitative realtime PCR experiments (Johnson et al., 2014). Specific primers were designed for each $M$. viciae OBP gene and for the reference genes, using Primer Express v3.0 software (ABI, Foster City, CA, United States). Primers of about $20 \mathrm{bp}$, with approximately $50 \%$ G/C content, were selected (Table 1). PCR amplifications were performed using GoTaq qPCR Master Mix (Promega, Madison, WI, United States). The reactions were carried out in a $20 \mu \mathrm{l}$ final volume containing $5 \mu \mathrm{l}$ of diluted first-strand cDNA $(60 \mathrm{ng} / \mu \mathrm{l})$ and $0.3 \mu \mathrm{mol} / \mathrm{L}$ primer final concentration. Cycling conditions for all genes were: $2 \mathrm{~min}$ at $95^{\circ} \mathrm{C}, 40$ cycles of $15 \mathrm{~s}$ at $95^{\circ} \mathrm{C}$ and $1 \mathrm{~min}$ at $60^{\circ} \mathrm{C}$. At the end of each run, a melting curve analysis was performed in order to confirm the specificity of PCR products. All amplification reactions were run in triplicate (technical replicates) and included negative controls (no template reactions, replacing cDNA with $\mathrm{H}_{2} \mathrm{O}$ ). All the experiments were performed for a set of 3 biological replicates. In order to evaluate gene expression levels, relative quantification was performed using the equations described by Liu and Saint (2002), based on PCR amplification efficiencies of reference and target genes. Amplification efficiency of each target gene and of RPS9 and RPL32 was determined according to the equation $E=10^{-1 / S}-1$ (Lee et al., 2006), where $S$ is the slope of the standard curve generated from 4 serial 10 -fold dilutions of cDNA.
TABLE 1 | Primers used for RT-qPCR.

\begin{tabular}{|c|c|}
\hline Gene Name & Primer sequence $\left(5^{\prime}-3^{\prime}\right)$ \\
\hline \multirow[t]{2}{*}{ MvicOBP1 } & F: ACCACATTGTTAACGACGGC \\
\hline & R: GTTGCGGCTAACTCACACTC \\
\hline \multirow[t]{2}{*}{ MvicOBP2 } & F: CCAAGCCAACAATGACCGAA \\
\hline & R: GCCTTCTTGTGTTCGTCTGG \\
\hline \multirow[t]{2}{*}{ MvicOBP3 } & F: CTAGGACTGCTGAACGACGA \\
\hline & R: CAGACATGCCATCACAGTGT \\
\hline \multirow[t]{2}{*}{ MvicOBP4 } & F: ACGTAGAGTTGCAGGGTGTT \\
\hline & R: TCGAAACTITGGAGGGCTG \\
\hline \multirow[t]{2}{*}{ MvicOBP5 } & F: AGTAGCAGCTGACGAGTGTT \\
\hline & R: CGTCTTCGGTGAGCAAATGA \\
\hline \multirow[t]{2}{*}{ MvicOBP6 } & F: GAAAAGAGCCACCATGTCTT \\
\hline & R: TTGGGGCAGCTCATATACAT \\
\hline \multirow[t]{2}{*}{ MvicOBP7 } & F: TTGCGACGCTTACTTGAGTG \\
\hline & R: TGTTGTTGTTGTCCTCCGGA \\
\hline \multirow[t]{2}{*}{ MvicOBP8 } & F: TGATGGGTTGCCTGATGAGA \\
\hline & R: AAGTTGTCACAATTCCGGCC \\
\hline \multirow[t]{2}{*}{ MvicOBP9 } & F: TGCCGGAGAAGAACTTGGAA \\
\hline & R: CCTTCAGTGCTGGTGATTCC \\
\hline \multirow[t]{2}{*}{ MvicOBP10 } & F: AGTGTTGCTTAGACGAGATGT \\
\hline & R: AACAAAAGCCGCTTCCAAAC \\
\hline \multirow[t]{2}{*}{ RPS9 } & F: TTCTGGGAGTCCAAACGAAC \\
\hline & R: TCTTGGAACGCAGACTTCAA \\
\hline \multirow[t]{2}{*}{ RPL32 } & F: ATGCTGCCTTCCAAATTCCG \\
\hline & R: ACGTGCATTTCCATTGGTCA \\
\hline
\end{tabular}

F, forward primer; $R$, reverse primer; RPS9, RPL32, reference genes.

All data (mean $\pm \mathrm{SD}$ ) were compared by one-way analysis of variance (ANOVA) and Tukey's HSD multiple comparisons test using GraphPad Prism 6.00 software for Windows (GraphPad Software, La Jolla, CA, United States ${ }^{1}$ ). Significant differences were expressed in terms of $p$-value $\left({ }^{*} p<0.05\right.$, ${ }^{* *} p<0.01$, $* * * p<0.001)$.

\section{Whole-Mount Immunolocalization Experiments}

For this assay, only the wingless morphs, collected at the first day of the adult stage, were considered. In particular, 6 antennae, 6 mouthparts, 6 cornicles and 6 caudae from wingless specimens were dissected under the microscope and washed twice with PBS, $\mathrm{pH}$ 7.4. Given that winged aphids are rare and difficult to recover and maintain in breeding they were not considered for this analysis. After the washing step, samples were fixed in $4 \%$ paraformaldehyde in PBS for $2 \mathrm{~h}$ and then washed twice with the same buffer. Samples were then incubated for $30 \mathrm{~min}$ with PBS containing $2 \%$ BSA (to reduce non-specific binding) and $0.1 \%$ of the detergent Tween 20 (Sigma) to permeabilize tissues favoring the entrance of antibodies. Samples were then incubated for $1 \mathrm{~h}$ at room temperature with antisera raised in rabbit, diluted 1:200. Whole mount immunolocalization experiments were carried out on five among the ten identified OBPs because only five antibodies were already available. We used antisera against OBPs

${ }^{1}$ www.graphpad.com 
$1,3,6,7$, and 8 of $A$. pisum since they are ortholog genes of M. viciae OBPs (Zhou et al., 2010). Antibodies, kindly provided by Prof. Paolo Pelosi (University of Pisa), were produced against the entire amino acid OBP sequences and they were not affinity purified. Since recombinant OBPs were not available for preadsorption controls against OBP antibodies, we validated their specificity by western blot using protein extract from the whole M. viciae body (Supplementary Figure S1). Briefly, we used $20 \mu \mathrm{g}$ of proteins (each lane), separated by a $12 \%$ polyacrylamide gel electrophoresis and transferred on a Whatman nitrocellulose membrane. Anti-OBP antibodies were diluted 1:1000 in trisbuffered saline and $0.1 \%$ Tween 20 (TBS-T) with 5\% bovine serum albumin (BSA). Goat anti-rabbit antibodies conjugated to horseradish peroxidase, diluted 1:15000 in TBS-T, was used as a secondary antibody after a pre-absorption using an extra lane loaded with protein extracted from aphid whole body. For detection, enhanced chemo luminescence (ECL) was used and signals were measured with Chemidoc ${ }^{\mathrm{TM}}$ MP System.

These antibodies have been previously used in experiments on the pea aphid $A$. pisum OBPs (De Biasio et al., 2015) and on the peach aphid Myzus persicae OBPs (Sun et al., 2013), that are orthologs of $A$. pisum OBPs (Zhou et al., 2010). We confirmed the high similarity level among $A$. pisum and $M$. viciae OBPs by amino acid alignment reported in Supplementary Figure S2.

Samples were washed with PBS and incubated for $1 \mathrm{~h}$ in a dark chamber with the secondary goat anti-rabbit tetramethylrhodamine (TRITC)-conjugated antibody diluted 1:200 (Jackson, Immuno Research Laboratories Inc., West Grove, PA, United States) in blocking solution containing $0.1 \%$ Tween 20 . In all controls, primary polyclonal anti-OBPs antibodies were omitted or substituted with rabbit pre-immune serum (1:200), and sections were treated with blocking solution containing $0.1 \%$ Tween 20 (Sigma) and incubated only with the secondary antibody. Coverslips were mounted with City fluor (City fluor Ltd., London, United Kingdom), and immunofluorescence was analyzed using an inverted laser-scanning confocal microscope (TCS SP5, Leica Microsystems, Wetzlar, Germany) equipped with a HCX PL APO lambda blue $63.0 \times 1.40$ NA OIL UV objective. Images were acquired using the Leica TCS software (emission windows fixed in the 551-626 range) without saturating any pixel. Z-stack sections acquisition was carried out by selecting the optimized acquisition parameters. The displayed bright field and fluorescent images represent Z-stack projections of sections obtained with the open source image software Fiji (average intensity) (Schindelin et al., 2012). Fluorescence and bright field images were combined with Adobe Photoshop (Adobe Systems Incorporated, San Jose, CA, United States).

\section{Behavioral Assays}

The behavioral response of $M$. viciae to the components of the alarm pheromone was investigated under the conditions reported in Sun Y.F. et al. (2012) for A. pisum, using a Y-tube. Briefly, an airflow of $0.5 \mathrm{~L} / \mathrm{min}$ was introduced into each arm of the glass Y-tube olfactometer through a glass stimulus chamber (odor source adapter) attached to each of the two arms. In each test, $1 \mu \mathrm{l}$ of hexane solution of each chemical compound, concentration $0.5 \%$, was placed in the glass stimulus chamber of the "treatment" arm. As a control, $1 \mu \mathrm{l}$ of hexane was placed in the glass stimulus chamber of the "control" arm of the olfactometer. Groups of twenty wingless adult aphids were introduced at the bottom of the Y-shaped copper wire and allowed to walk to either arm at the Y-junction. After $15 \mathrm{~min}$, the number of aphids in the treatment and control sides of the olfactometer were counted. Six replications with each compound were performed. Tested compounds were (E)- $\beta$-farnesene (Bedoukian Research, Danbury, CT, United States), ( \pm )- $\alpha$-pinene, $\beta$-pinene, (-)$\alpha$-pinene, $(+)$-limonene, hexane (Sigma-Aldrich-Fluka) and a mixture comprising (E)- $\beta$-farnesene $14.2 \%$, (-)- $\alpha$-pinene $11.8 \%$ and $\beta$-pinene $74 \%$ (Francis et al., 2005). The behavioral responses to all the analyzed compounds and mixture were compared by one-way analysis of variance (ANOVA) and Tukey's HSD multiple comparisons test using GraphPad Prism 6.00 software for Windows (GraphPad Software) $\left({ }^{*} p<0.05,{ }^{* *} p<0.01\right.$, $* * *$ $p<0.001)$.

\section{RESULTS}

\section{Scanning Electron Microscopy of Sensilla}

Scanning electron microscopy observations of $M$. viciae highlighted differences of legs and antennae both in the morphology and in the distribution of sensilla (Supplementary Figures S3A-D and Figures 1A-N). In legs, numerous trichoid sensilla, uniform in size, shape and distribution, were visible. In the vetch aphid, sensilla showed a peak with a rounded shape, without pores (Supplementary Figures S3A,B,D). SEM images show the insertion of the sensillum basal portion in a cuticular extension on the leg (Supplementary Figure S3C). On the antennae of both wingless (Figures $\mathbf{1 A - N}$ ) and winged morph (Supplementary Figures S4A-H), different types of sensilla were recognizable, depending on the segment. Type II trichoid sensilla were located on the antenna tip of the 6th segment and along the processus terminalis on the same segment. Type II trichoid sensilla located on the antenna tip appeared as short hairs with a blunt tip showing fissure-like structures and grooves (Figures 1A,B and Supplementary Figures S4A,A'). Type II trichoid sensilla on the processus terminalis (Figures $\mathbf{1 C}, \mathbf{C}$ ' and Supplementary Figures S4B,B'), and type I trichoid sensilla, visible from the base of the antenna to the 6th segment, were characterized by a grooved surface and a swollen tip with fissure-like and porous structures (Figures 1D-F,I,J,L,M and Supplementary Figures S4C,F,F,G,H). Primary rhinaria were clearly observable on the 5th and 6th antennal segments (Figure 1D and Supplementary Figures S4C,F). In particular, a placoid sensillum was located in the distal end of the 5th segment (Figures 1D,I and Supplementary Figure S4F), while on the 6th segment 1 large placoid sensillum, 2 smaller ones, 2 type I and 2 type II coeloconic sensilla were distinguishable and surrounded by cuticular fringes (Figures 1D,E,G,H and Supplementary Figures S4C-E). The placoid sensilla appeared as circular plates showing porous structures on their flat surface (Figures 1E,I,K and Supplementary Figures S4E,F). On the 3rd antennal segment, secondary rhinaria were constituted by about 
30 placoid sensilla in the wingless aphids (Figure 1L) and of about 60 placoid sensilla in the winged morph (Supplementary Figures S4G,H), both showing a smooth ridge not surrounded by cuticular fringes and small pores on their flat surface (Figure 1N). Moreover, we found that in the winged aphids the $3 \mathrm{rd}$ segment was longer than in wingless morph $(1040 \mu \mathrm{m}$ instead of $743 \mu \mathrm{m}$ ). Both the wingless and winged vetch aphid presented sensilla associated with mouthparts and caudal region. Since no differences between the two morphs were found, only data of winged morph were shown (Figures 2A-I). In the mouthparts, these sensilla showed different morphologies: they had pre-lobed apical extensions (Figure 2B) or branched tips (Figure 2C). Numerous short sensilla, arranged symmetrically, were evident on the labium end part (Figure 2A). SEM observations of the cauda (Figures 2D-F) showed the presence of long sensory hair-like structures with small pores (Figure 2E) or a fissure-like structure (Figure 2F). The entire caudal surface was covered by numerous finger-like projections arranged in groups (Figure 2D). Similar structures were also found on the surface of cornicles (Figures 2G,H). In addition, the terminal region of cornicles was characterized by the presence of cuticular fingers among which holes were visible (Figure 2I).

Scanning electron microscopy observations of $M$. viciae legs and antennae highlighted differences both in the morphology and in the distribution of sensilla. In legs, numerous trichoid sensilla were visible. On the antennae of both wingless and winged morph type II trichoid sensilla, type I trichoid sensilla, primary rhinaria (5th and 6th segments) and secondary rhinaria (3rd segment) were found. Moreover, the vetch aphid presented sensilla associated with mouthparts and caudal region.

\section{Identification of OBP Candidates}

First, putative OBP coding sequences needed to be identified. To this end, RNA sequencing of $M$. viciae antennae was performed. Sequencing data were assembled using the Trinity assembler, resulting in 43,251 predicted transcripts from 36,239 'genes'. The N50 of the assembled transcripts was 2,063 bp, with a corresponding median contig length of $571 \mathrm{bp}$, average of $1,115 \mathrm{bp}$ and $48,243,578$ total nucleotides in the assembly. The assembled data were used in the identification and annotation of ten candidate OBP genes, named MvicOBP1, MvicOBP2, MvicOBP3, MvicOBP4, MvicOBP5, MvicOBP6, MvicOBP7, MvicOBP8, MvicOBP9, and MvicOBP10. The nucleotide sequences were deposited in GenBank under the accession numbers listed in Table 2. OBPs expression level in antennae was estimated as reads per kilobase per million mapped reads (RPKM).

Among the ten identified candidate OBP genes, MvicOBP1, MvicOBP3, MvicOBP6, MvicOBP7 and MvicOBP8 were selected for immunolocalization analysis because antibodies were already available. Antibodies against $A$. pisum OBPs were used because of the high sequence similarity among the selected $M$. viciae OBPs and the same A. pisum OBPs (Supplementary Figure S2). The alignment of the 10 identified antennal $M$. viciae OBPs is shown in Supplementary Figure S5.

RNA sequencing and assembly of $M$. viciae antennae allowed the identification and the annotation of ten candidate OBP genes.
MvicOBP1, MvicOBP3, MvicOBP6, MvicOBP7, and MvicOBP8 were selected for immunolocalization analysis because specific antibodies were already available.

\section{OBP Expression Patterns in Different Body Parts and Nymphal Instars of M. viciae}

In order to evaluate the expression level in different body parts of all the ten identified $M$. viciae OBPs, RT-qPCR experiments were carried out using gene-specific primers and using RPS9 and $R P L 32$ as reference genes. We validated the use of these reference genes in RT-qPCR experiments on different developmental stages of M. viciae, in a previous work (Cristiano et al., 2016) and we repeated the validation step on the analyzed different body parts observing that the expression levels of RPS9 and RPL32 remained the same (Supplementary Figure S6). Supplementary Figure S7 shows the OBPs relative expression calibrated on RPS9 and $R P L 32$, respectively. RT-qPCR results showed that $M v i c O B P 1$ and $M v i c O B P 10$ transcripts were significantly more expressed in $M$. viciae antennae than in the other body parts $\left({ }^{* * *} p<0.001\right)$. Transcripts coding for MvicOBP2 were more expressed in antennae, cauda and bodies than in heads and legs $(* * p<0.01)$, while transcripts for $M v i c O B P 3$ were significantly more expressed in antennae $\left({ }^{*} p<0.05\right)$ and in cauda $\left({ }^{* *} p<0.01\right)$. For MvicOBP4 the statistically highest transcript levels were observed in antennae and bodies (**p $<0.01$ ), while the expression levels of MvicOBP5 were statistically the same in antennae, cauda, bodies and legs $\left({ }^{*} p<0.05\right)$. For MvicOBP6 and MvicOBP7, the statistically highest transcript expression levels were observed in antennae $\left({ }^{* *} p<0.01\right)$ and in heads $\left({ }^{* *} p<0.01\right.$ for MvicOBP6 and ${ }^{*} p<0.05$ for MvicOBP7). Moreover, we found that the gene encoding for $M v i c O B P 8$ was statistically mainly expressed in the cauda and in heads $\left({ }^{* *} p<0.01\right)$, while MvicOBP9 transcripts were more expressed in antennae $\left({ }^{* *} p<0.01\right)$ and heads $\left({ }^{*} p<0.05\right)$ (Figure 3 ).

RT-qPCR experiments were confirmed by whole-mount immunolocalization experiments carried out on five OBPs for which antibodies were available (Figure 4). In particular, MvicOBP1, MvicOBP3, MvicOBP6, and MvicOBP7 were immunolocalized in type II trichoid sensilla (Figures 4A-I) and in the primary rhinaria located on the 5th and 6th segments of antenna (Figures $4 \mathbf{K}-\mathbf{S}$ ). MvicOBP1 was expressed mainly in the lymph of type I trichoid sensilla located on the 6th segment (Figures $\mathbf{4 F - K}$ ). Moreover, MvicOBP1 was expressed on placoid sensilla located on the 3rd, 5th, and 6th antennal segments (Figures 4K,P,U). MvicOBP3 was expressed in the lymph of type II trichoid sensilla located on the distal region of the antenna (Figures $\mathbf{4 B}, \mathbf{G}$ ) and in the large placoid sensilla on the 6th segment (Figures 4L). Moreover, MvicOBP3 was expressed in placoid sensilla on the 5th and 3rd segments (Figures 4Q,V). In contrast, the small placoid sensilla and the coeloconic sensilla on the 6th segment were not labeled by the antiserum against MvicOBP3 (Figure 4L). MvicOBP6 was immunolocalized in the lymph of all sensilla located on the 3rd, 5th, and 6th antennal segments, except in type I trichoid sensilla, and in the 6th segment coeloconic sensilla 

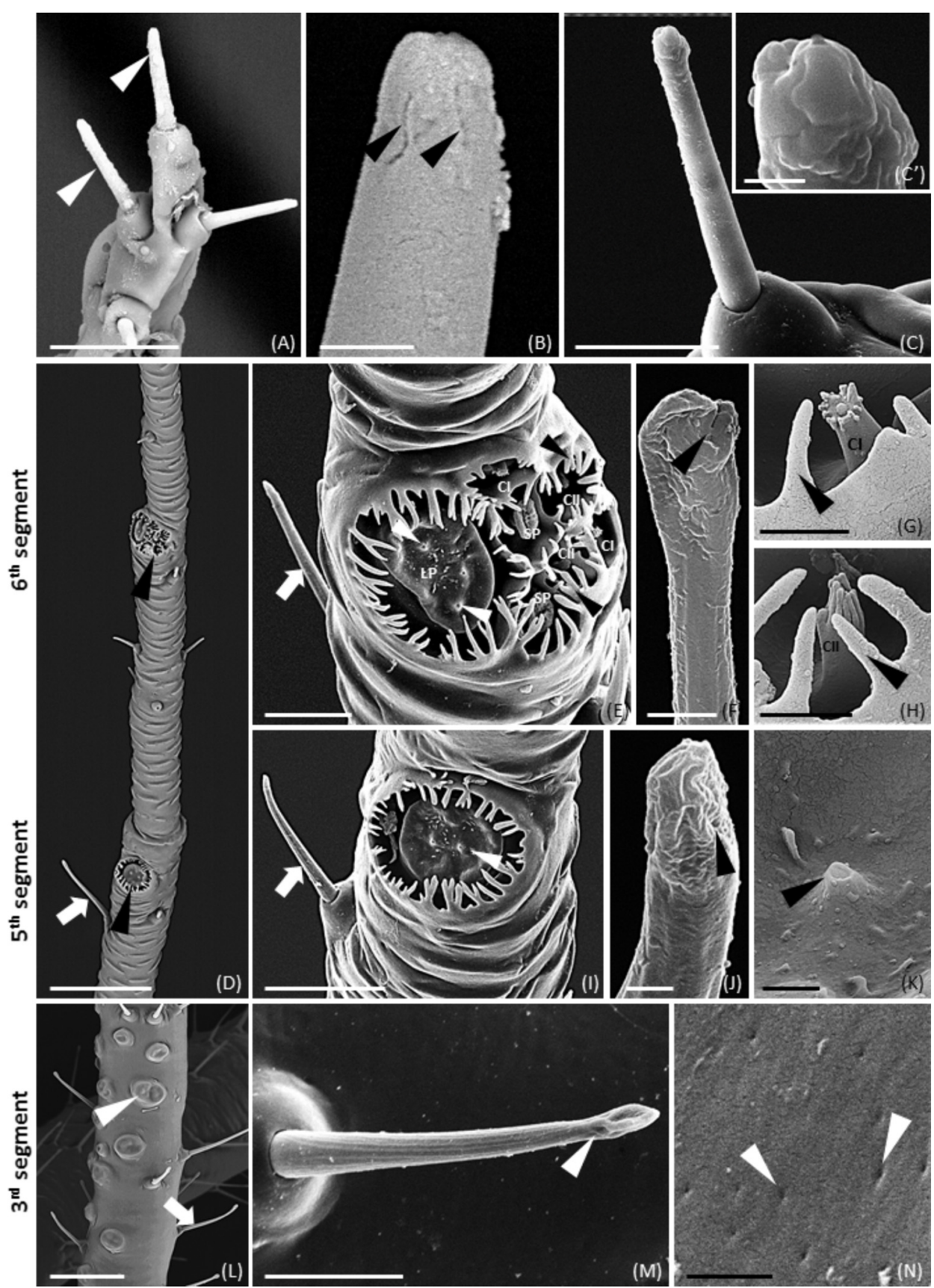

FIGURE 1 | SEM images showing the distribution and morphology of different sensilla on wingless M. viciae antennae. (A-C) Type II trichoid sensilla located on the terminal part of the antenna (arrowheads in (A)), and on processus terminalis (C) showing a blunt tip with a grooved surface (B,C'). (D) Global view of primary rhinaria on 5 and 6th segments (arrowheads) with a type I trichoid sensilla (arrow). (E) Details of the primary rhinaria on the 6th segment composed of 1 large placoid sensillum (LP) with porous structures (white arrowheads), 2 small placoid sensilla (SP), and 4 coeloconic pegs surrounded by cuticular fringes (black arrowheads). $(\mathbf{G}, \mathbf{H})$ Detail of type I (Cl in (G)) and type II (CII in (H)) coeloconic sensilla in the 6th segment surrounded by cuticular fringes (arrowheads). (I) Detail of placoid sensillum of 5th segment. Porous structures were visible on the flat surface (arrowhead in (K)). (L) Placoid sensilla forming the secondary rhinaria of the 3rd segment (arrowhead) and trichoid sensilla (arrow). (F,J,M) Details of type I trichoid sensilla showing a groove surface and porous structures on the tip. (N) Detail of a placoid sensillum with a smooth surface not surrounded by cuticular fringes and small pores on the flat surface. Bars in (A,E,I,M), $10 \mu \mathrm{m}$; bars in (B,F-H,K), $1 \mu \mathrm{m}$; bar in (C), $5 \mu \mathrm{m}$; bars in (C',J,N), $500 \mathrm{~nm}$; bars in (D,L), $50 \mu \mathrm{m}$.

(Figures 4C,H,M,R,W). Finally, placoid and trichoid sensilla on the 3rd and 5th segments and the lymph of type II trichoid sensilla, placoid and coeloconic sensilla on the 6th segment were labeled specifically by the antibody against $M v i c O B P 7$, while type I trichoid sensilla on the 6th segment were not stained by this antibody (Figures 4D,I,N,S,X). In none of sensilla described 

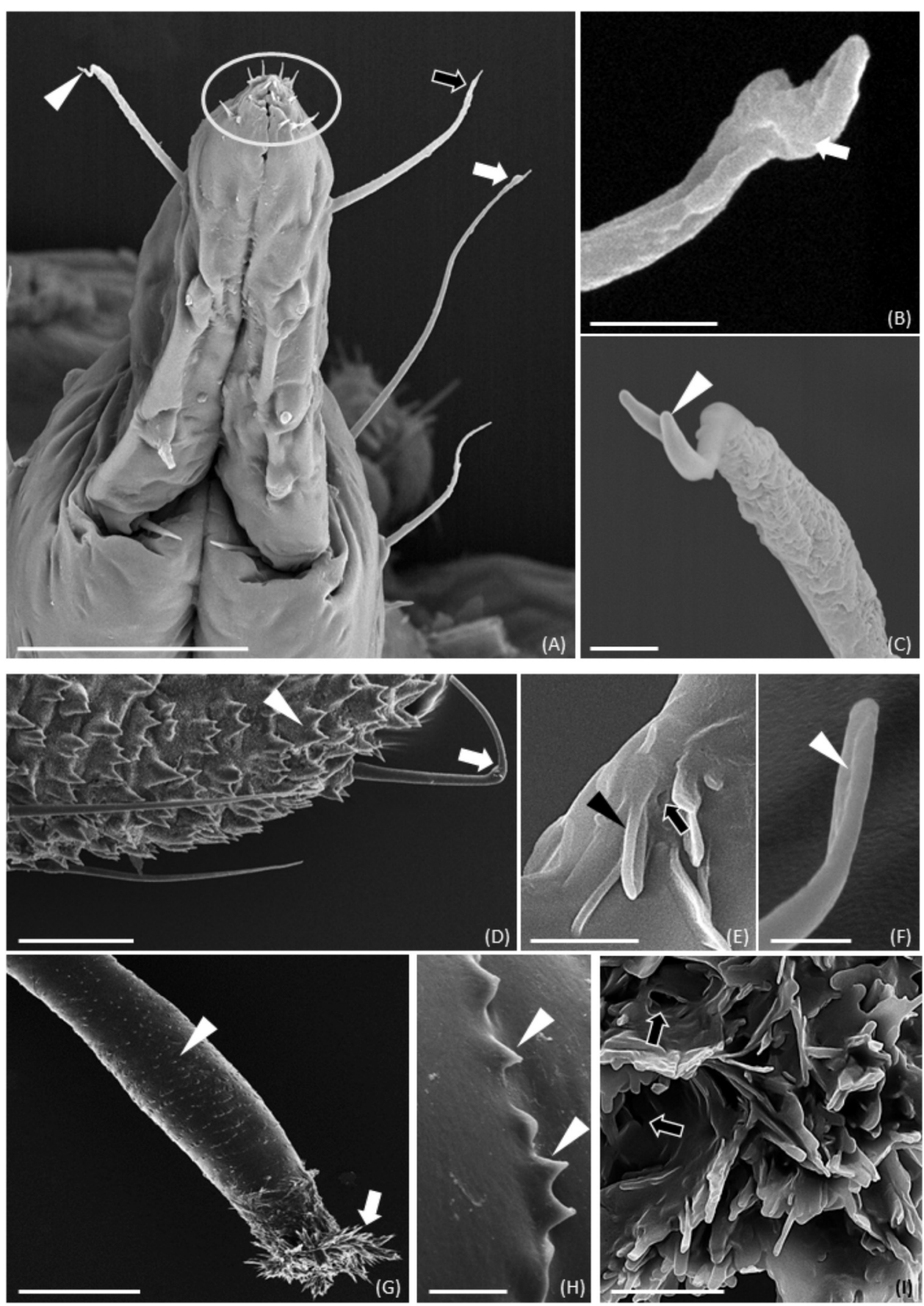

FIGURE 2 | SEM images showing sensilla on M. viciae mouthparts, cauda and cornicles. (A) Long sensilla symmetrically distributed (arrowhead) and short sensilla (encircled) situated on the labium tip. (B) Detail of long sensilla tip with pre-apical expansion (black arrow in (A)) or in the shape of a cup (arrow in (A,B)). (C) Detail of long sensilla branched tip (arrowhead). (D-F) Detail of porous (arrow in (E)) or fissure like structures (arrowheads in (E,F)) on long sensilla and finger-like extensions (arrowhead in (D)) on M. viciae cauda. Finally, SEM observation highlights the presence of cuticular finger-like structures (arrowheads in (G,H)) on cornicle surface. Moreover, hole-like structures are evident among cuticular tufts (arrows in (I)) of cornicle terminal region. Bar in (A), $50 \mu \mathrm{m}$; bars in (B,E,F), $1 \mu \mathrm{m}$; bar in (C), $2 \mu \mathrm{m}$; bar in (D), $25 \mu \mathrm{m}$; bar in (G), $100 \mu \mathrm{m}$; bars in (H,I), $5 \mu \mathrm{m}$.

above, we found the expression of MvicOBP8 (Supplementary Figure S8A). The expression profile of OBPs in the mouthparts (Figure $\mathbf{4 A}^{\prime}$ ) and in the terminal body part (Figure $\mathbf{4 B}^{\prime}$ ) is shown in Figures $4 \mathbf{C}^{\prime}-\mathbf{E}^{\prime}, \mathbf{G}^{\prime}-\mathbf{L}^{\prime}$. In the mouthparts, MvicOBP6,
MvicOBP7 and MvicOBP8 were expressed in the inner lymph of hair-like sensilla (Figures $4 \mathbf{C}^{\prime}-\mathbf{E}^{\prime}$ ). In contrast, no signal was detected for MvicOBP1 and MvicOBP3 (Supplementary Figures S8C,F). MvicOBP3 and MvicOBP8 were detected in 
TABLE 2 | Candidate OBP genes in Megoura viciae antennae.

\begin{tabular}{|c|c|c|c|c|c|c|c|}
\hline $\begin{array}{l}\text { Unigene } \\
\text { reference }\end{array}$ & Gene name & ORF (bp) & $\begin{array}{l}\text { Accession } \\
\text { number }\end{array}$ & BLASTx annotation & $E$-value & $\begin{array}{l}\text { AA Identity } \\
(\%)\end{array}$ & $\begin{array}{l}\text { Antennae } \\
\text { RPKM value }\end{array}$ \\
\hline 4148_c0_g2_i1 & MvicOBP1 & 480 & MG596881 & $\begin{array}{l}\text { [NP_001153526.1] Odorant-binding protein } 1 \\
\text { precursor [Acyrthosiphon pisum] }\end{array}$ & $2 e-111$ & 99 & 3.81028 \\
\hline 3537_c0_g1_i1 & MvicOBP2 & 726 & MH177887 & $\begin{array}{l}\text { [NP_001153528.1] Odorant-binding protein } 2 \\
\text { precursor [Acyrthosiphon pisum] }\end{array}$ & $5 e-165$ & 95 & 7.56772 \\
\hline 20255_c0_g1_i1 & MvicOBP3 & 426 & MG596882 & $\begin{array}{l}\text { [NP_001153529.1] Odorant-binding protein } 3 \\
\text { precursor [Acyrthosiphon pisum] }\end{array}$ & $4 e-92$ & 96 & 4.57565 \\
\hline 10025_c0_g1_i1 & MvicOBP4 & 600 & MH177888 & $\begin{array}{l}\text { [NP_001153530.1] Odorant-binding protein } 4 \\
\text { precursor [Acyrthosiphon pisum] }\end{array}$ & $7 e-128$ & 93 & 5.65031 \\
\hline 5845_c0_g1_i1 & MvicOBP5 & 666 & MH177889 & $\begin{array}{l}\text { [NP_001153531.1] Odorant-binding protein } 5 \\
\text { precursor [Acyrthosiphon pisum] }\end{array}$ & $3 e-152$ & 95 & 6.1596 \\
\hline 9875_c1_g1_i3 & MvicOBP6 & 648 & MG596883 & $\begin{array}{l}\text { [NP_001153532.1] Odorant-binding protein } 6 \\
\text { [Acyrthosiphon pisum] }\end{array}$ & $8 e-104$ & 95 & 2.75666 \\
\hline 5098_c0_g1_i1 & MvicOBP7 & 468 & MG596884 & $\begin{array}{l}\text { [NP_001153533.1] Odorant-binding protein } 7 \\
\text { precursor [Acyrthosiphon pisum] }\end{array}$ & $4 e-96$ & 88 & 3.92025 \\
\hline 18200_c0_g1_i1 & MvicOBP8 & 486 & MG596885 & $\begin{array}{l}\text { [NP_001153534.1] Odorant-binding protein } 8 \\
\text { precursor [Acyrthosiphon pisum] }\end{array}$ & $2 e-96$ & 95 & 4.3177 \\
\hline 594_c0_g1_i1 & MvicOBP9 & 501 & MH177890 & $\begin{array}{l}\text { [NP_001153535.1] Odorant-binding protein } 9 \\
\text { precursor [Acyrthosiphon pisum] }\end{array}$ & $7 e-102$ & 90 & 6.26894 \\
\hline 23913_c0_g1_i1 & MvicOBP10 & 435 & MH177891 & $\begin{array}{l}\text { [NP_001153525.1] Odorant-binding protein } 10 \\
\text { [Acyrthosiphon pisum] }\end{array}$ & $8 e-58$ & 81 & 5.65906 \\
\hline
\end{tabular}

the hair-and finger-like structures of the terminal region of the body and in the cornicles (Figures 4G'-L'), while in both these regions no signals were found for MvicOBP1, MvicOBP6, MvicOBP7 (Supplementary Figures S8D,E,G-J). No signal was detected in control experiments in which the primary antibodies were substituted with the rabbit pre-immune serum (Figures 4E,J,O,T,Y,F',M',N') or omitted (Supplementary Figure S8B).

Table 3 summarizes the localization of the five analyzed MvicOBPs in different sensilla types in the wingless morph.

RT-qPCR was also used to investigate on the OBPs expression levels in different nymphal instars. Results showed that $M v i c O B P 1$ transcripts were significantly more expressed in the IV nymphal instar $\left.{ }^{* * *} p<0.001\right)$, in the winged adults $\left({ }^{* *} p<0.01\right)$ and both in the wingless adults and III nymphal instar $\left({ }^{*} p<0.05\right)$. MvicOBP2 transcripts were significantly more expressed in the winged morph $\left({ }^{* *} p<0.01\right)$. Transcripts encoding for MvicOBP3 showed high expression levels in the IV nymphal instar and in the wingless adults $\left({ }^{* *} p<0.01\right)$, which agrees with the lower levels of expression observed in the early nymphal instars $\left({ }^{*} p<0.05\right)$ and in the winged adults. MvicOBP4 transcripts were more expressed in the II and IV nymphal instar $\left({ }^{* *} p<0.01\right)$, while expression of MvicOBP5 was statistically higher only in the IV nymphal instar (** $p<0.01)$. MvicOBP6 transcripts were found to be more expressed in the early nymphal instars (I, II, III) $\left({ }^{*} p>0.05\right)$. Equally, the levels of transcription of the gene encoding for $M v i c O B P 8$ were statistically higher in the first two pre-productive stages (I and II) and in the winged adult morph $\left({ }^{*} p>0.05\right)$. The expression of the gene encoding for MvicOBP7 was higher both in the II and IV nymphal instar and in the wingless adult stage $(* p>0.05)$, but lower in the other immature stages (I, III) and in winged. Equally, transcripts encoding for MvicOBP9 were more expressed in the IV instar $\left({ }^{* *} p<0.01\right)$ and in the II and wingless morph $\left({ }^{*} p<0.05\right)$. The expression of the gene encoding for MvicOBP10 was higher both in the IV nymphal instar $\left({ }^{*} p<0.05\right)$ and in winged adult $\left(*^{* *} p<0.01\right)$ (Figure 5).

All the ten identified MvicOBPs were analyzed by RT-qPCR in different body parts and in all the developmental stages. MvicOBP1, MvicOBP3, MvicOBP6, MvicOBP7 and MvicOBP8 were selected for further analysis of immunolocalization showing a complex immunolocalization pattern in all the analyzed body parts (antennae, mouthparts, cornicles and cauda).

\section{Behavioral Experiments}

Behavioral experiments on $M$. viciae wingless adults were performed with the main compounds identified in a cornicle droplet ((E)- $\beta$-farnesene, $\beta$-pinene, $(-)-\alpha$-pinene and $(+)$ limonene). For the experiments, a Y-tube olfactometer was used, and aphids that did not choose either of the two arms of the olfactometer (chemical or solvent) were not included in the analysis. The repellency $(\mathrm{R})$ of each compound was calculated by the formula $R=(\mathrm{C}-\mathrm{T}) /(\mathrm{C}+\mathrm{T})$, where $\mathrm{T}$ indicates the number of aphids in the arm with the compound to be tested, and $\mathrm{C}$ indicates the number of aphids in the control arm. A value of $R=1$ indicates that all the insects that have chosen were found in the control arm, while $R=0$ indicates that as the aphids were distributed equally between the two arms, the tested substance clearly had no effect. Results are shown in Figure 6. The aphids were repelled significantly by $(-)$ - $\alpha$-pinene, $(+)$ limonene and the mixture containing (E)- $\beta$-farnesene $14.2 \%$, (-)- $\alpha$-pinene $11.8 \%, \beta$-pinene $74 \%$ (Francis et al., 2005), with the $R$-values of $0.40,0.28$ and 0.48 , respectively. In contrast, $( \pm)-\alpha-$ pinene, $\beta$-pinene and (E)- $\beta$-farnesene alone were not repellent for $M$. viciae, with the $R$-values of $0.07,-0.05$ and 0.02 , respectively (Figure 6). 

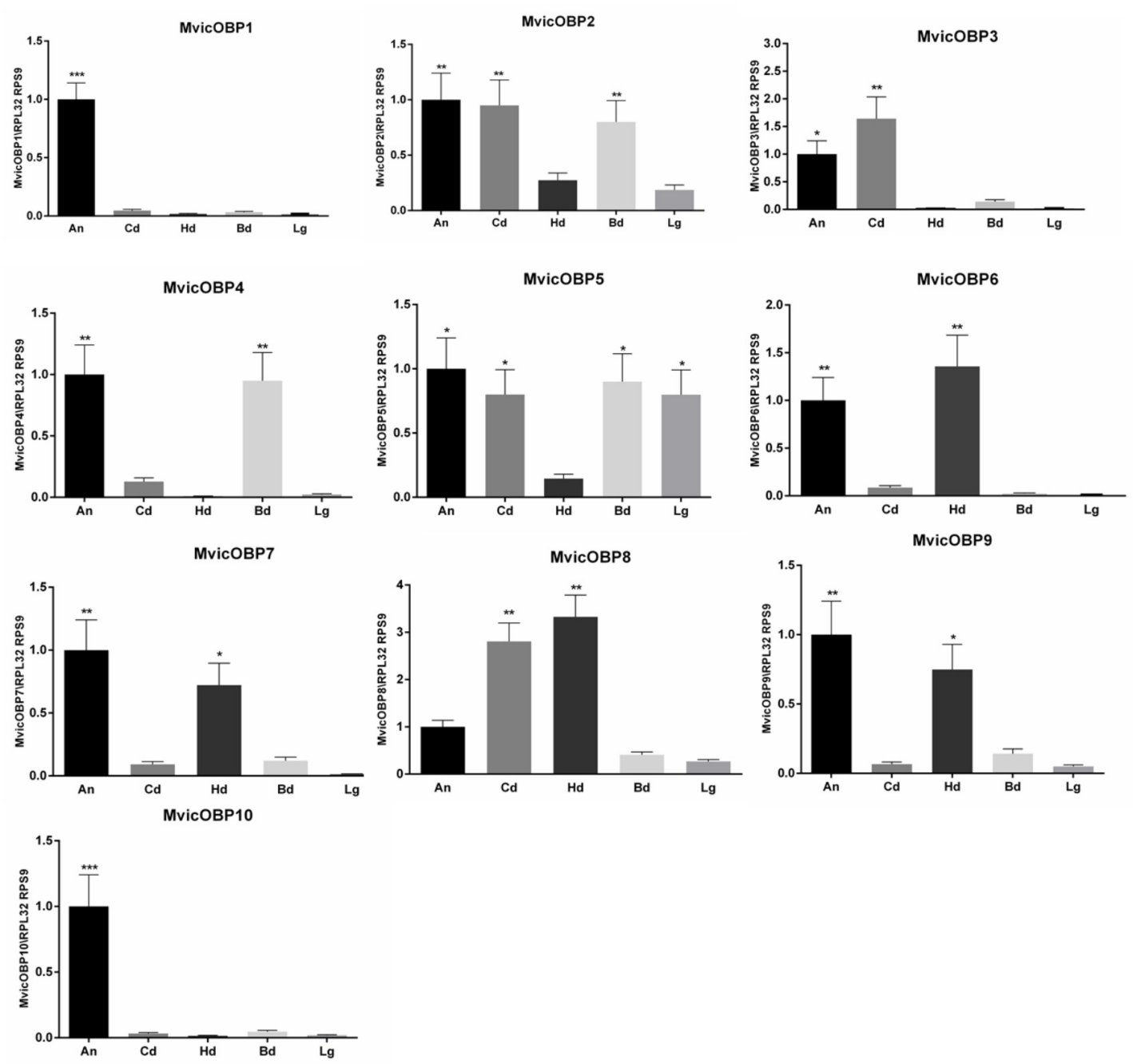

FIGURE 3 | Relative expression level of $M$. viciae OBPs in different body parts. OBP expression levels were quantified by RT-qPCR. Bars represent the standard deviation of the mean for 3 independent experiments. Significant differences are denoted by asterisks (Tukey's test, $\left.\left({ }^{*} p<0.05, * * p<0.01, * * * p<0.001\right)\right)$. Lg, legs; Cd, cornicles-cauda; Hd, head; Bd, body; An, antennae. Reference genes: RPL32, RPS9. Calibrator sample: antennae.

Behavioral experiments on M. viciae unwinged adults were performed with the main compounds identified in a cornicle droplet. Aphids were repelled significantly by $(-)-\alpha$-pinene, $(+)$-limonene and the mixture containing (E)- $\beta$-farnesene, (-)$\alpha$-pinene and $\beta$-pinene.

\section{DISCUSSION}

Odorant-binding proteins are classically defined as olfactory soluble proteins (Vogt et al., 1991; Pelosi, 1994). Since OBPs are expressed in organs devoted to chemoreception, such as antennae and mouthparts, they likely play a role related to chemoreception. The fact that OBPs are expressed in sensilla whose cuticular surface allows the entry of molecules able to stimulate the olfactory and gustatory receptors located on the sensory neurons strengthens this likelihood (Diehl et al., 2003;
De Biasio et al., 2015; Pelosi et al., 2017). Considering that OBPs are also expressed in several organs not related to olfactory and gustatory perception, they can conceivably perform different functions (Nomura et al., 1992; Kitabayashi et al., 1998).

In addition, the same OBP can perform different roles when expressed in different organs and tissues which is related to the general ability of OBPs to bind and transport a range of small molecules, not only those deriving from the external environment (Jacquin-Joly et al., 2001; Zhou et al., 2004; Smartt and Erickson, 2009; Strandh et al., 2009; Sun Y.L. et al., 2012; Gu et al., 2013; Ishida et al., 2013; Zhang et al., 2013, 2015; Xia et al., 2015; Pelosi et al., 2017).

Although it is now generally recognized that OBPs are involved in cellular processes other than chemoreception, the important role of OBPs in chemoreception is confirmed. These soluble proteins, by binding small hydrophobic molecules, allow their solubilization in the sensory lymph (carrier role) and at 

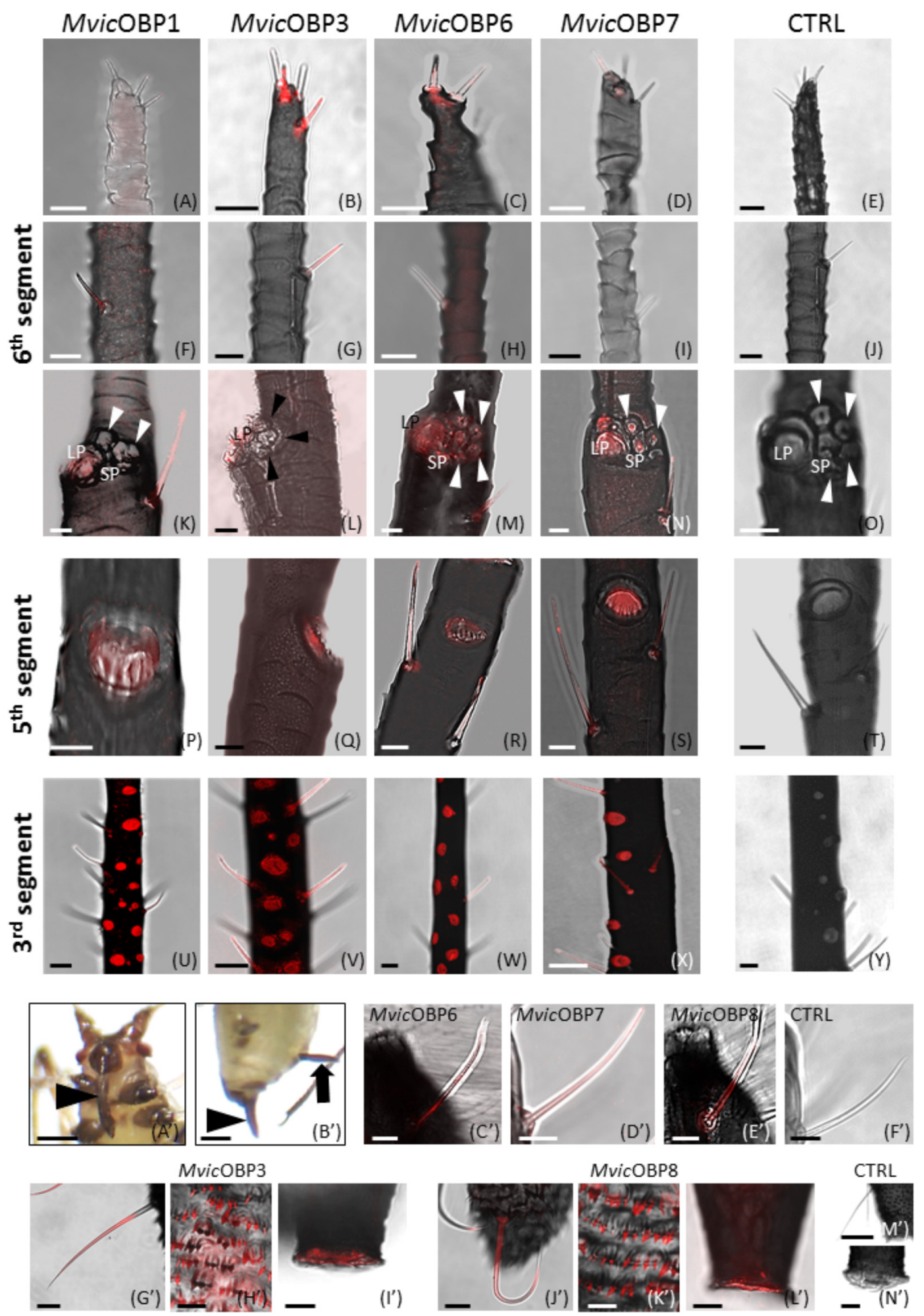

MvicOBP8
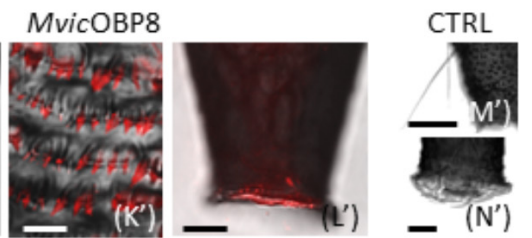

FIGURE 4 | (A-Y) Whole-mount immunolocalization experiments showing the OBP expression in type II trichoid sensilla located on the antennal tip (A-D), in type II trichoid sensilla on the 6th antennal segment (F-I), in primary rhinaria on the 5th (K-N) and 6th segments (P-S) and in secondary placoid sensilla on the 3rd segment (U-X). (E,J,O,T,Y) Negative controls. Bars in (A-T), $10 \mu \mathrm{m}$; bars in (U-Y), $25 \mu \mathrm{m}$. ('A'-N') Whole-mount immunolocalization experiments showing the OBP

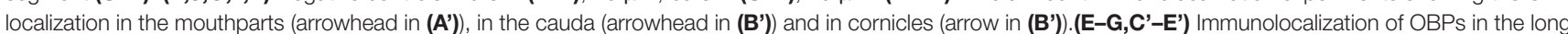
sensilla on the labium sides. (G'-L') OBPs detection in hair-like structures and finger-like projections in cauda and in cornicles. (F',M',N') Negative controls. Bars in ('A',B'), $250 \mu \mathrm{m}$; bars in (C'-F',H',K'), $10 \mu \mathrm{m}$; bars in (M'), $50 \mu \mathrm{m}$; bars in (G',l',J',L',N'), $20 \mu \mathrm{m}$ 
TABLE 3 | Immunolocalization of five among the ten identified MvicOBPs in different body parts.

\begin{tabular}{|c|c|c|c|c|}
\hline & \multicolumn{4}{|c|}{ Immunolocalization } \\
\hline & Antennae & Mouthparts & Cauda & Cornicles \\
\hline MvicOBP1 & $\begin{array}{l}\text { - Type II trichoid sensilla, } 5 \text { and } 6 \text { th segments } \\
\text { - Type I trichoid sensilla, 6th segment } \\
\text { - Primary rhinaria } \\
\text { - Secondary rhinaria }\end{array}$ & None & None & None \\
\hline MvicOBP3 & $\begin{array}{l}\text { - Type II trichoid sensilla, antennal tip } \\
\text { - Type II trichoid sensilla, } 5 \text { and 6th segments } \\
\text { - Primary rhinaria } \\
\text { - Secondary rhinaria }\end{array}$ & None & $\begin{array}{l}\text { Hair- and finger-like structures } \\
\text { of the terminal region }\end{array}$ & Detected \\
\hline MvicOBP6 & $\begin{array}{l}\text { - Type II trichoid sensilla, antennal tip } \\
\text { - Type II trichoid sensilla, } 5 \text { and 6th segments } \\
\text { - Primary rhinaria } \\
\text { - Secondary rhinaria }\end{array}$ & Hair-like sensilla & None & None \\
\hline MvicOBP7 & $\begin{array}{l}\text { - Type II trichoid sensilla, } 5 \text { and 6th segments } \\
\text { - Primary rhinaria } \\
\text { - Secondary rhinaria }\end{array}$ & Hair-like sensilla & None & None \\
\hline MvicOBP8 & None & Hair-like sensilla & $\begin{array}{l}\text { Hair- and finger-like structures } \\
\text { of the terminal region }\end{array}$ & Detected \\
\hline
\end{tabular}

the same time the protection against degradation performed by odorant degrading enzymes (ODEs) and the increase of sensitivity toward the receptors (Gomez-Diaz et al., 2013; Chertemps et al., 2015). In this work, we focused on the ten OBPs identified as transcripts in the aphid Megoura viciae antennae. Since the sensilla type and morphology provides an indication about the attribution of a hypothetical functional role of the OBPs expressed therein, an integrated and multidisciplinary approach has been adopted, starting from the analysis of the antennal ultrastructure in both wingless and winged adult morphs and on the different types of sensilla, through SEM.

Two types of trichoid sensilla (I and II) have been described in M. viciae adults (wingless and winged) as in other aphid species (Bromley et al., 1980; Sun et al., 2013; De Biasio et al., 2015). Four type II trichoid sensilla, with a blunt tip characterized by the presence of fissure-like structures are located on the aphid antenna distal part on the 6th segment, both in wingless and winged morphs. These fissure-like structures described for the first time on the type II trichoid sensilla at the end of the processus terminalis would appear similar to those found in the pea aphid A. pisum on the long hair tip of the mouthparts (De Biasio et al., 2015). In A. pisum, the inner lymph of fissured hair like sensilla on the mouthparts was immunostained by the antibody against an ApisOBP (ApisOBP8). Similarly, in M. viciae lymph of fissured type II trichoid sensilla on the antenna tip is immunostained by antibodies against MvicOBP3, MvicOBP6, MvicOBP7. The immunolocalization of all these OBPs and the simultaneous presence of fissure-like structures suggest that fissures on these types of sensilla might be involved in the entry of chemical molecules.

Otherwise, in $M$. viciae, type II trichoid sensilla located along the processus terminalis and type I trichoid sensilla visible along the whole length of the antennae are characterized by the presence of apical and longitudinal grooves similar to those observed in other insect species (Diehl et al., 2003; Palma et al., 2013; Missbach et al., 2014) where these sensilla were described as olfactory sensilla. They are morphologically different from the same category of sensilla observed in the two aphid species, A. pisum (De Biasio et al., 2015) and M. persicae (Sun et al., 2013), where a smooth surface and a rounded tip have been described. It is interesting to observe that type I trichoid sensilla in $M$. viciae are stained by antibodies against MvicOBP1, MvicOBP3, MvicOBP6, MvicOBP7, which is in contrast to A. pisum and $M$. persicae in which type I trichoid sensilla were not stained by any anti-OBP antibody, and for which a mechanoreceptive function was hypothesized (Shambaugh et al., 1978; Bromley et al., 1979; Sun et al., 2013; De Biasio et al., 2015). A possible role of $M$. viciae type I and type II trichoid sensilla in chemical perception could be hypothesized on the basis of immunolocalization signals and on the basis of the observed morphology that at the ultrastructural level highlights the presence of grooves.

Moreover, SEM observations show the presence of a single large placoid sensillum, two smaller placoid sensilla and four coeloconic sensilla (type I and II) located on the 6th segment, and a single large placoid sensillum on the 5th segment of both wingless and winged adults, as already described for A. pisum and for other species of aphids (Shambaugh et al., 1978; Bromley et al., 1979; Sun et al., 2013; De Biasio et al., 2015). Already available data describing the ultrastructure of aphid placoid sensilla show the localization of pore structures on these sensilla surface (Bromley et al., 1979; Sun et al., 2013). Pore like structures have been observed also in Megoura viciae placoid sensilla indicating that they could be typical chemosensilla as demonstrated in other aphids (Wohlers and Tjallingii, 1983; Park and Hardie, 2004). In primary rhinaria (5th and 6th segments) differences between wingless and winged adults concerning shape, distribution and number of placoid sensilla have not been observed. Secondary rhinaria on the $3 \mathrm{rd}$ antennal segment in $M$. viciae are constituted by placoid sensilla too, similar in the general morphology to those found in the 5th and the 6th segments, suggesting a shared chemosensory function. In winged M. viciae morph, about 60 

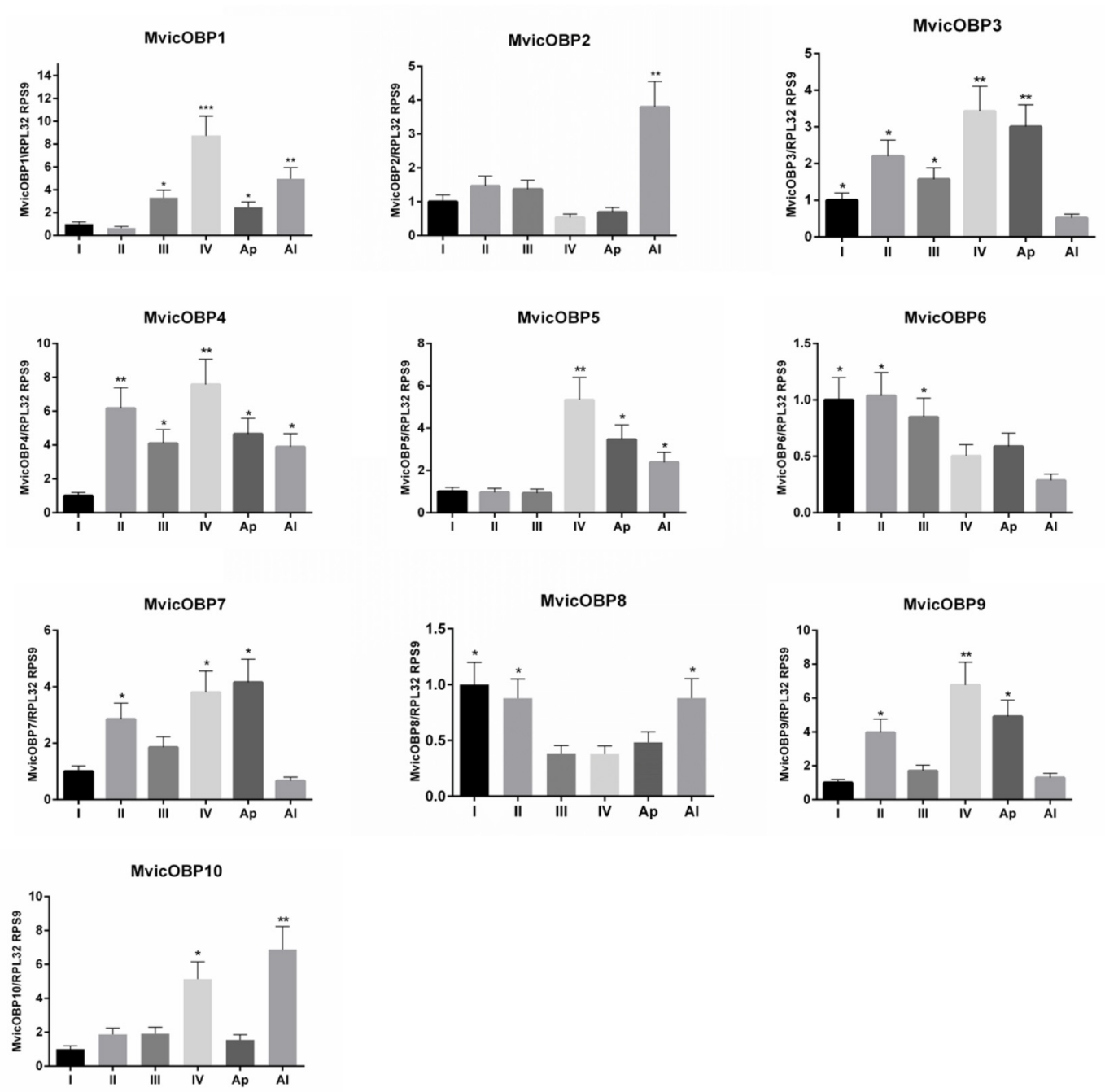

FIGURE 5 | Relative expression level of $M$. viciae OBPs in different nymphal instars. OBP expression levels were quantified by RT-qPCR. Bars represent the standard deviation of the mean for 3 independent experiments. Significant differences are denoted by asterisks (Tukey's test, $\left({ }^{*} p<0.05,{ }^{* *} p<0.01,{ }^{* * *} p<0.001\right)$ ). I, 1 st nymphal instar; II, 2nd nymphal instar; III, 3rd nymphal instar; IV, 4th nymphal instar; Ap, winged adults; Al, winged adults. Reference genes: RPL32, RPS9.

Calibrator sample: 1st nymphal instar

placoid sensilla on the 3rd segment have been counted, whereas about 30 placoid sensilla have been counted in wingless insects on the same segment. In addition, the length of the 3rd segment increases by about $40 \%$ in winged adults. These differences suggest a potential involvement of these sensilla in the location of new host plants. Indeed, aphids acquire wings only when they need to change host plant or mate; therefore, these sensilla could be involved in the detection of plant volatiles (Pickett et al., 1992; Sun et al., 2013).

MvicOBP1, MvicOBP3, MvicOBP6, MvicOBP7 have been immunolocalized in the lymph of placoid sensilla on the 3rd and 5th segments and in large placoid sensilla on the 6th aphid antennal segment. RT-qPCR data confirm the immunolocalization signals of $M v i c O B P 1$ showing that the relative expression of this OBP is significantly higher in the antennae. The immunolocalization pattern of MvicOBP6 follows what had been already described in A. pisum in which OBP6 was immunolocalized in placoid sensilla (large and small) on the 6th segment, in placoid sensilla on the 5th segment and in secondary rhinaria (De Biasio et al., 2015). RT-qPCR data confirm the immunolocalization signals, showing that the relative expression of MvicOBP6 is significantly higher in the antennae. EAG experiments performed on different aphid species demonstrate that primary rhinaria (both proximal and distal) are able to perceive a range of plant volatiles. More specifically, the distal primary rhinaria (DPR) are significantly more responsive to tested alcohols than aldehydes in comparison to the proximal primary rhinaria (PPR) and vice versa, indicating a difference in the perception of plant volatiles between the two primary rhinaria (Pickett et al., 1992; van Giessen et al., 1994). Behavioral and electrophysiological studies demonstrated that secondary rhinaria in $M$. viciae and in other aphids are responsive to sex 


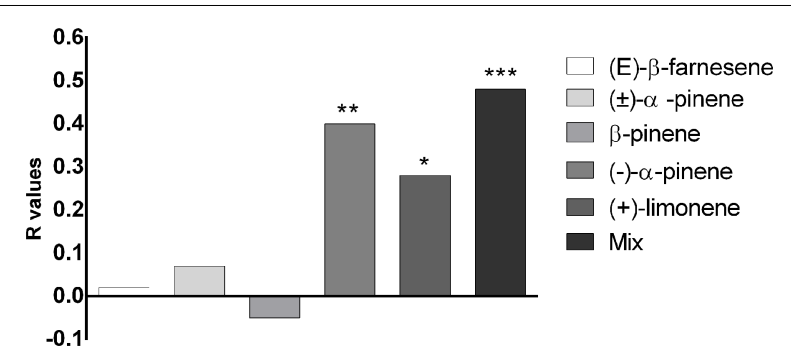

FIGURE 6 | Behavioral responses of $M$. viciae to the main compounds identified in the insect's cornicle secretions and to the mixture containing (E)- $\beta$-farnesene $14.2 \%,(-)$ - $\alpha$-pinene $11.8 \%, \beta$-pinene $74 \%$. The repellency index $R$ was calculated by the formula $R=(C-T) /(C+T)$, where $T$ indicates the number of aphids in the arm with the compound to be tested and $\mathrm{C}$, those in the control arm. Asterisks indicate that the repellence observed is significantly different from the control $\left({ }^{*} p<0.05,{ }^{* *} p<0.01,{ }^{* * *} p<0.001\right.$, Student's $t$-test)

pheromone (Pettersson, 1971; Marsh, 1975; Dawson et al., 1987, 1988; Campbell et al., 1990). The immunolocalization signals of MvicOBP1 and MvicOBP6 both in primary and secondary rhinaria and the significantly high relative expression level of these OBPs in the antennae suggest a possible involvement of MvicOBP1 and MvicOBP6 in the perception of host plant chemical volatiles and sex pheromones.

RT-qPCR data also confirm the immunolocalization signals of $M v i c O B P 3$ and $M v i c O B P 7$ showing that the relative expression of these OBPs is higher in the antennae. A. pisum and M. persicae OBP3 and OBP7, orthologs of M. viciae (Zhou et al., 2010), have high binding affinity to the (E)- $\beta$-farnesene (EBF) which is the only component of the alarm pheromone in these two aphid species. The alarm pheromone triggers physiological and behavioral responses in the aphid colony, to stimulate conspecifics to leave the host plant immediately (Sun Y.F. et al., 2012). In $M$. persicae and A. pisum, OBP7 was immunolocalized in the primary rhinaria of the 5th and the 6th segments (PPR and DPR), but only in $M$. persicae OBP7 was also localized in the secondary rhinaria of the 3 rd segment. M. persicae OBP3, on the other hand, was immunolocalized in the PPR and only low signals were detected in the other placoid sensilla (Sun et al., 2013) whereas ApisOBP3 was exclusively expressed in the DPR (De Biasio et al., 2015). It had been demonstrated that in A. pisum the perception of EBF involves only primary rhinaria and more specifically DPR, totally excluding secondary rhinaria. Similarly, in the vetch aphid Megoura viciae, EBF is exclusively perceived by DPR (Wohlers and Tjallingii, 1983). Nevertheless, MvicOBP3 and MvicOBP7 have been immunolocalized both in primary (distal and proximal) and secondary rhinaria, unlike ApisOBP3 and ApisOBP7. This may seem surprising but it is conceivable that the involvement of at least $M v i c O B P 3$ in the perception of the other components of the alarm pheromone, as previously demonstrated (Northey et al., 2016), may take place in sensilla different from primary rhinaria. Indeed, it has been demonstrated that different OBPs can bind the same molecules in a single organism (Sun Y.F. et al., 2012). Likewise, orthologous OBPs can bind the same molecules in different organisms (Sun Y.F. et al., 2012; Northey et al., 2016) but also different molecules in different organisms (Northey et al., 2016).

Immunolocalization experiments localize $M v i c O B P 3$ also in cornicles and cauda, which is confirmed at the mRNA level by RT-qPCR results. This finding does not represent an absolute novelty, since OBP3 expression in A. pisum, evaluated by RTqPCR and immunolocalization, was also observed in cornicles and cauda (De Biasio et al., 2015). The authors hypothesized that ApisOBP3 could be involved in the transport of the alarm pheromone EBF to the environment. Indeed, aphid cornicles are involved in the release of liquid substances in response to dangerous situations such as the presence of predators or parasitoids (Capinera, 2008). The fluid is composed of the alarm pheromone and of other lipid compounds, such as triglycerides, with sticky properties able to trap natural enemies (Strong, 1967; Callow et al., 1973; Greenway and Griffiths, 1973; Butler and O'Neil, 2006; van Emden and Harrington, 2007; De Biasio et al., 2015). Since it has been demonstrated that MvicOBP3 binds EBF and other components of the alarm pheromone mixture (Northey et al., 2016), it is reasonable to suppose that $M v i c O B P 3$, expressed in the cornicles, on which hole-like structures are evident, could be involved in the transport of the alarm pheromone mixture to the environment, suggesting also in this species that OBPs could perform roles other than chemoreception.

The alarm pheromone covers an important physiological role in aphids and its use has been proposed in the development of potential strategies for aphid population control (Sun et al., 2011). The identification of OBPs able to bind this pheromone with high affinity is therefore particularly relevant. Although in most aphid species, including A. pisum, the major component of alarm pheromone is the EBF, in $M$. viciae the alarm pheromone is composed by a mixture of different compounds, including EBF (Bowers et al., 1972; Edwards et al., 1973; Pickett and Griffiths, 1980; Francis et al., 2005). It was demonstrated that ApisOBP3, ApisOBP7 and ortholog proteins have high binding affinity for EBF (Sun Y.F. et al., 2012; Zhang et al., 2017). MvicOBP3 binds EBF with high affinity but it was not able to bind the other components of the alarm pheromone $((-)$ - $\alpha$-pinene, $\beta$-pinene, $(+)$-limonene) with the same affinity (Northey et al., 2016). The evaluation of the contribution of each component and the mixture to aphids repulsion behavior is required to address the identification of $M v i c O B P s$ binding these components. As expected, the mix of (E)- $\beta$-farnesene, $(-)$ - $\alpha$-pinene, $\beta$-pinene and $(+)$-limonene is significantly more repellent in comparison to the effect of the single components. Surprisingly, (E)- $\beta$-farnesene alone, as well as $\beta$-pinene alone and the racemic mixture $( \pm)$ - $\alpha$-pinene, is not active against $M$. viciae. The most active single components are $(-)-\alpha$-pinene and (+)-limonene. The behavioral assay represents the basis to address the identification and functional characterization of MvicOBPs directly involved in mediating $M$. viciae dispersion behavior.

MvicOBP8 is expressed in cornicles and in cauda long sensilla, where pores and fissure like structures have been observed, as well as in finger-like extensions that cover the entire cauda surface, 
different to what has been described for A. pisum (De Biasio et al., 2015). RT-qPCR data confirm the immunolocalization of MvicOBP8, showing that this OBP is significantly expressed in cornicles and cauda. It is interesting to note that, similarly to A. pisum OBPs, also M. viciae OBPs, such as MvicOBP8 in this case, are expressed in organs apparently not related to chemoreception, such as the finger-like extensions on the cauda, suggesting a possible new function that needs to be further investigated.

In insects in general and in aphids in particular, other organs besides the antennae are related to chemoreception. SEM revealed that both the wingless and the winged vetch aphid present sensilla associated with mouthparts. Immunolocalization experiments performed on the mouthparts show that the lymph of these sensilla are stained with MvicOBP6, MvicOBP7 and MvicOBP8 antibodies. RT-qPCR data confirm the immunolocalization signals of these OBPs, showing also that the relative expression levels are significantly higher in heads. In accordance with what had been already observed in A. pisum, whose OBP8 was immunolocalized in the sensilla on the mouthparts (De Biasio et al., 2015), MvicOBP8 is immunolocalized in the long sensilla located on the lateral part of the labium. However, unlike what had been observed in A. pisum, OBP6 and OBP7 in Megoura viciae are found in the long hair sensilla. The observed expression patterns suggest that the three OBPs could cover a task in gustatory perception. Indeed, plant volatiles and non-volatiles (such as alkaloids and terpenoids) are moderately soluble in water and the three OBPs may be involved also in the interaction with hydrophobic non-volatile molecules (Galindo and Smith, 2001; Jeong et al., 2013; Swarup et al., 2014), suggesting a greater complexity in the mechanisms of chemoreception also involving $M$. viciae mouthparts.

Numerous trichoid sensilla have been found on the whole surface of the leg. These types of sensilla are uniform in size, shape and distribution and are similar to those already described in A. pisum (De Biasio et al., 2015). RT-qPCR shows a very low expression level for all the analyzed OBPs, with the exception of MvicOBP5, and no signal in the immunolocalization experiments.

All the results obtained by RT-qPCR experiments on the OBPs whose antibodies were already available are consistent with the results obtained by immunolocalization. We have thus carried out RT-qPCR experiments also on the other OBPs identified in the transcriptome (MvicOBP2, MvicOBP4, MvicOBP5, MvicOBP9, MvicOBP10), for which immunolocalization experiments have not been possible since no specific antibodies were available. All OBPs show significantly higher relative expression levels in the antennae compared to the other organs tested, allowing to hypothesize a possible role in chemoreception for these OBPs. MvicOBP2 and MvicOBP5 show a similar expression pattern, except for the higher relative expression level of MvicOBP5 in legs. MvicOBP5 is the only OBP among those identified in the transcriptome that is significantly expressed in the legs. Since in this aphid species the sex pheromone is produced and released at numerous plaques localized on the hind tarsi, a potential role for MvicOBP5 in sex pheromone release and/or interaction can be speculated. Different roles were attributed to these organs on hind tarsi and it was suggested that they produce a sex pheromone able to attract male aphids (Flogejl, 1905; Weber, 1935; Smith, 1936; Bodenheimer and Swirski, 1957; Stroyan, 1958; Pettersson, 1971; Marsh, 1972, 1975). MvicOBP9 show a relative expression pattern similar to MvicOBP6 and MvicOBP7. Although it was not possible to evaluate the immunolocalization for this OBP, the similar expression profile suggests an analogous function. Similarly, we hypothesize that MvicOBP10 may be involved in a task analogous to that covered by $M v i c O B P 1$ in the light of the very similar expression pattern.

MvicOBP3, MvicOBP5, MvicOBP7 and MvicOBP9 are most highly expressed in IV nymphal instar and wingless morph. The observed higher expression levels of these two OBPs could relate to a higher necessity of these later developmental stages to perceive certain compounds (Roitberg and Meyers, 1978) when compared to lower transcript levels in the early stages. MvicOBP1 displays the highest expression levels in IV nymphal instar and winged adults while MvicOBP2, MvicOBP8 and MvicOBP10 are primarily expressed in the winged morph. Moreover, MvicOBP6 is mostly expressed in the first nymphal instars while MvicOBP4 is expressed in the first nymphal instars and in the more mature instars (including the winged morph). The marked heterogeneity of our M. viciae OBPs expression level analysis at different developmental stages could be explained with the complexity of the molecular mechanisms that drive the behavioral response of the different aphids' nymphal instars to the chemical molecules. Indeed, different plant chemicals are able to trigger different behavioral responses that are also dependent on aphid morph and developmental stage; moreover, different morphs of the same aphid species show different behaviors in response to the same volatiles (Lilley and Hardie, 1996; Quiroz and Niemeyer, 1998; Powell and Hardie, 2001; Webster, 2012). Within the same morph, the response to volatile compounds can vary widely in relation to the stage of development (Glinwood and Pettersson, 2000a,b).

\section{CONCLUSION}

In this work we have verified which of the identified OBPs were expressed in sensilla that, for their position in typical chemoreceptive organs and for the presence of morphological features such as pores, grooves and fissure-like structures, could potentially cover chemoreceptive functions. Considering the traditional role attributed to OBPs, the gained information would have led us to assign automatically a specific role of odorants carrier toward the olfactory receptors to the identified OBPs. In the light of recent works (e.g., Larter et al., 2016) the OBPs expressed in chemosensilla are certainly involved in chemoreception but their roles can be multiple, although the specific feature of binding proteins remains unaltered (Pelosi et al., 2017). Our data on the ultrastructure of sensilla as well as on OBP expression profiles in different developmental stages and various body parts allow to state that OBPs in Megoura viciae show a very complex expression pattern. The increasing 
knowledge about the different tasks performed by OBPs in insects leads us to hypothesize that the described level of complexity of Megoura viciae OBPs pattern can be ascribed to the different functions of these proteins in physiological pathways of the vetch aphid. The knowledge acquired with this work could represent the road map for guiding future studies aimed to the detailed clarification of the role of each $M$. viciae OBP.

\section{DATA AVAILABILITY STATEMENT}

The raw data supporting the conclusions of this manuscript will be made available by the authors, without undue reservation, to any qualified researcher.

\section{AUTHOR CONTRIBUTIONS}

$\mathrm{PF}$ designed the experiments, and wrote and critically revised the paper. EG-W, HV, BH, J-JZ, AS, GG, RS, and SB contributed to the data interpretation and critically revised the paper. AG and DB performed the SEM experiments and immunolocalization experiments. GG, DF, and RS performed the samples collection, RT-qPCR, and antibodies validation. GG and AS performed the behavioral assays. J-JZ performed the antennal transcriptome sequencing. GG, HV, and EG-W performed the transcriptome analysis. All authors read and approved the manuscript.

\section{FUNDING}

This work was supported by the Max Planck Society and by University of Basilicata (RIL funds).

\section{ACKNOWLEDGMENTS}

$\mathrm{DB}$ is a student of the Ph.D. program in Biotechnology, Biosciences and Surgical Technologies, School in Biological and Medical Sciences, University of Insubria. We would like to thank Emily Wheeler for editorial assistance, Silvia Sacchi and Marcella Reguzzoni for their technical assistance for SEM and confocal image analysis and Vincenzo Trotta for the assistance in statistical analysis.

\section{REFERENCES}

Ban, L. P., Napolitano, E., Serra, A., Zhou, X., Iovinella, I., and Pelosi, P. (2013). Identification of pheromone-like compounds in male reproductive organs of the oriental locust Locusta migratoria. Biochem. Biophys. Res. Commun. 437, 620-624. doi: 10.1016/j.bbrc.2013.07.015

Biessmann, H., Andronopoulou, E., Biessmann, M. R., Douris, V., Dimitratos, S. D., Eliopoulos, E., et al. (2010). The Anopheles gambiae odorant binding protein 1 (AgamOBP1) mediates indole recognition in the antennae of female mosquitoes. PLoS One 5:e9471. doi: 10.1371/journal.pone.000 9471

Bodenheimer, F. S., and Swirski, E. (1957). The Aphidoidea of the Middle East. Jerusalem: Weizmann Science Press of Israel.

\section{SUPPLEMENTARY MATERIAL}

The Supplementary Material for this article can be found online at: https://www.frontiersin.org/articles/10.3389/fphys. 2018.00777/full\#supplementary-material

FIGURE S1 | Western blot performed with antisera against OBPs 1, 3, 6, 7, and 8 of $A$. pisum on protein extract from the whole $M$. viciae body ( $20 \mu \mathrm{g}$ of proteins per each lane).

FIGURE S2 | Alignment of amino acid sequences of Megoura viciae and Acyrthosiphon pisum OBPs

FIGURE S3 | SEM images showing the distribution of sensilla on M. viciae legs. Trichoid sensilla present a typical hair shape and are covered by a thin cuticle (arrowheads in (A-C)). These sensilla show a peak with a rounded shape, without pores. Bar in (A), $100 \mu \mathrm{m}$; bar in (B), $25 \mu \mathrm{m}$; bar in (C), $100 \mu \mathrm{m}$; bar in (D), $2 \mu \mathrm{m}$

FIGURE S4 | SEM images showing the distribution and morphology of different sensilla on winged $M$. viciae antennae. $(\mathbf{A}, \mathbf{B})$ Type II trichoid sensilla located on the terminal part of the antenna (arrowhead in (A)) and on processus terminalis (B) with grooves on tip surface (arrowhead in (A', $\left.\mathbf{B}^{\prime}\right)$ ). (C) Global view of primary rhinaria on 6th segment (arrowhead) and type II trichoid sensilla (arrow). (D) Details of small placoid sensilla (SP), and type I (Cl) and type II (CII) coeloconic sensilla in the 6th segment surrounded by cuticular fringes (arrowheads). (E) Detail of porous structure on the surface of the large placoid sensillum (arrowheads). (F) Details of placoid sensillum of 5 th segment and type I trichoid sensilla (arrow) with grooved surface (arrow in $\left(\mathbf{F}^{\prime}\right)$ ). Porous structures were visible on the flat surface on the placoid sensillum of this segment (arrowhead). (G,H) Placoid sensilla (secondary rhinaria) on the 3rd segment (white arrowhead in $(\mathbf{G}, \mathbf{H})$ ) and trichoid sensilla type I (arrow in (G,H)). Bars in (A,C,F), $10 \mu \mathrm{m}$; bars in (A', $\left.\mathbf{B}^{\prime}, \mathbf{F}^{\prime}\right), 500 \mathrm{~nm}$; bar in (B), $2 \mu \mathrm{m}$; bars in (D,E), $2 \mu \mathrm{m}$; bar in (G), $100 \mu \mathrm{m}$; bar in (H), $20 \mu \mathrm{m}$.

FIGURE S5 | Alignment of amino acid sequences of candidate OBPs from Megoura viciae.

FIGURE S6 | RPS9 and RPL32 constant expression level in M. viciae body parts.

FIGURE S7 | Relative expression level of $M$. viciae OBPs in different body parts $(\mathbf{A}, \mathbf{B})$ and in different nymphal instars (C,D) calibrated on RPL32 and RPS9, respectively. OBP expression levels were quantified by RT-qPCR. Bars represent the standard deviation of the mean for 3 independent experiments. Significant differences are denoted by asterisks (Tukey's test, $\left({ }^{*} p<0.05,{ }^{* *} p<0.01\right.$, $\left.\left.{ }^{* * *} p<0.001\right)\right)$. (A,B) Lg, legs; Cd, cornicles-cauda; Hd, head; Bd, body; An, antennae. Calibrator sample: antennae. (C,D) I, 1st nymphal instar; II, 2nd nymphal instar; III, 3rd nymphal instar; IV, 4th nymphal instar; Ap, apterous adults; Al, alata adults. Calibrator sample: 1 st nymphal instar.

FIGURE S8 | Whole-mount immunolocalization experiments showing the absence of signal for MvicOBP8 in antenna (A), MvicOBP1 and MvicOBP3 in the mouthparts (C,F), MvicOBP1, MvicOBP6, MvicOBP7 in the cauda (D,G,I), and in cornicles $(\mathbf{E}, \mathbf{H}, \mathbf{J})$. (B) Negative control in which the primary antibodies were

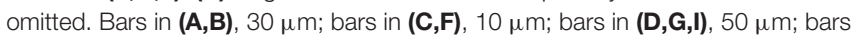
in $\mathbf{( E , H , J ) , ~} 20 \mu \mathrm{m}$.

Bowers, W. S., Nault, L. R., Webb, R. E., and Dutky, S. R. (1972). Aphid alarm pheromone: isolation, identification, synthesis. Science 177, 1121-1122. doi: $10.1126 /$ science.177.4054.1121

Brito, N. F., Moreira, M. F., and Melo, A. C. A. (2016). A look inside odorantbinding proteins in insect chemoreception. J. Insect Physiol. 95, 51-65. doi: 10.1016/j.jinsphys.2016.09.008

Bromley, A. K., Dunn, J. A., and Anderson, M. (1979). Ultrastructure of the antennal sensilla of aphids. I. Coeloconic and placoid sensilla. Cell Tissue Res. 203, 427-442. doi: 10.1007/BF00233272

Bromley, A. K., Dunn, J. A., and Anderson, M. (1980). Ultrastructure of the antennal sensilla of aphids. II. Trichoid, chordotonal and campaniform sensilla. Cell Tissue Res. 205, 493-551. doi: 10.1007/BF002 32289 
Bustin, S. A., Benes, V., Garson, J. A., Hellermans, J., Huggett, J., and Kubista, M. (2009). The MIQE guidelines: minimum information for publication of quantitative real-time PCR experiments. Clin. Chem. 55, 611-622. doi: 10.1373/ clinchem.2008.112797

Butler, C. D., and O'Neil, R. J. (2006). Defensive response of soybean aphid (Hemiptera : Aphididae) to predation by insidious flower bug (Hemiptera: Anthocoridae). Ann. Entomol. Soc. Am. 99, 317-320. doi: 10.1603/00138746(2006)099[0317:DROSAH]2.0.CO;2

Callow, R. K., Greenway, A. R., and Griffiths, D. C. (1973). Chemistry of the secretion from the cornicles of various species of aphids. J. Insect Physiol. 19, 737-748. doi: 10.1016/0022-1910(73)90146-7

Calvello, M., Guerra, N., Brandazza, A., D’Ambrosio, C., Scaloni, A., Dani, F. R., et al. (2003). Soluble proteins of chemical communication in the social wasp Polistes dominulus. Cell Mol. Life Sci. 60, 1933-1943. doi: 10.1007/s00018-0033186-5

Campbell, C. A. M., Dawson, G. W., Griffiths, D. C., Petterson, J., and Pickett, J. A. (1990). Sex attractant pheromone of damson- hop aphid Phorodon humili (Homoptera: Aphididae). J. Chem. Ecol. 26, 601-609. doi: 10.1007/BF009 82110

Capinera, J. L. (2008). Encyclopedia of Entomology, 2nd Edn, Vol. 1-4. Dordrecht: Springer, 4346. doi: 10.1007/978-1-4020-6359-6

Chertemps, T., Younus, F., Steiner, C., Durand, N., Coppin, C. W., Pandey, G., et al. (2015). An antennal carboxylesterase from Drosophila melanogaster, esterase 6, is a candidate odorant-degrading enzyme toward food odorants. Front. Physiol. 6:315. doi: 10.3389/fphys.2015.00315

Cristiano, G., Grossi, G., Scala, A., Fanti, P., Zhou, J. J., Bufo, S. A., et al. (2016). Validation of reference genes for qRT-PCR analysis in Megoura viciae (Hemiptera Aphididae). Bull. Insectol. 69, 229-238.

Dani, F. R., Michelucci, E., Francese, S., Mastrobuoni, G., Cappellozza, S., La Marca, G., et al. (2011). Odorant-binding proteins and Chemosensory proteins in pheromone detection and release in the silkmoth Bombyx mori. Chem. Senses 36, 335-344. doi: 10.1093/chemse/bjq137

Dawson, G. W., Griffiths, D. C., Janes, N. F., Mudd, A., Pickett, J. A., and Wadhams, L. J. (1987). Identification of an aphid sex pheromone. Nature 325, 614-616. doi: $10.1038 / 325614 \mathrm{a} 0$

Dawson, G. W., Griffiths, D. C., Merritt, L. A., Mudd, A., Pickett, J. A., Wadhams, L. J., et al. (1988). The sex pheromone of the greenbug, Schizaphis graminum. Entomol. Exp. Appl. 48, 91-93. doi: 10.1111/j.1570-7458.1988.tb 02303.x

De Biasio, F., Riviello, L., Bruno, D., Grimaldi, A., Congiu, T., Sun, Y. F., et al. (2015). Expression pattern analysis of odorant-binding proteins in the pea aphid Acyrthosiphon pisum. Insect Sci. 22, 220-234. doi: 10.1111/1744-7917. 12118

Diehl, P. A., Vlimant, M., Guerenstein, P., and Guerin, P. M. (2003). Ultrastructure and receptor cell responses of the antennal grooved peg sensilla of Triatoma infestans (Hemiptera: Reduviidae). Arthropod. Struct. Dev. 31, 271-285. doi: 10.1016/S1467-8039(03)00004-5

Distler, P. G., and Boeckh, J. (1996). Synaptic connection between olfactory receptor cells and uniglomerular projection neurons in the antennal lobe of the American cockroach, Periplaneta americana. J. Comp. Neurol. 370, 35-46. doi: 10.1002/(SICI)1096-9861(19960617)370:1<35::AID-CNE4>3.0.CO;2-M

Edwards, L. J., Siddall, J. B., Dunham, L. L., Uden, P., and Kislow, C. J. (1973). Trans- betafarnesene, alarm pheromone of green peach aphid, Myzus persicae (Sulzer). Nature 241, 126-127. doi: 10.1038/241126b0

Fan, J., Francis, F., Liu, Y., Chen, J. L., and Cheng, D. F. (2010). An overview of odorant-binding protein functions in insect peripheral olfactory reception. Genet. Mol. Res. 10, 3056-3069. doi: 10.4238/2011.December.8.2

Flogejl, H. L. (1905). Monographie der Johannisbeeren-Blattlaus, Aphis ribis L. Zeitschrift für wissenschaftliche Insektenbiologie 1, 49-63.

Francis, F., Vandermoten, S., Verheggen, F., Lognay, G., and Haubruge, E. (2005). Is the (E)- $\beta$-farnesene only volatile terpenoid in aphids? J. Appl. Entomol. 129, 6-11. doi: 10.1111/j.1439-0418.2005.00925.x

Galindo, K., and Smith, D. P. (2001). A large family of divergent Drosophila odorant-binding proteins expressed in gustatory and olfactory sensilla. Genetics 159, 1059-1072.

Glinwood, R. T., and Pettersson, J. (2000a). Change in response of Rhopalosiphum padi spring migrants to the repellent winter host component methyl salicylate. Entomol. Exp. Appl. 94, 325-330. doi: 10.1046/j.1570-7458.2000.00634.x
Glinwood, R. T., and Pettersson, J. (2000b). Host choice and host leaving in Rhopalosiphum padi (Hemiptera: Aphididae) emigrants and repellency of aphid colonies on the winter host. B Entomol. Res. 90, 57-61.

Gomez-Diaz, C., Reina, J. H., Cambillau, C., and Benton, R. (2013). Ligands for pheromone-sensing neurons are not conformationally activated odorant binding proteins. PLoS Biol. 11:e1001546. doi: 10.1371/journal.pbio.1001546

Grabherr, M. G., Haas, B. J., Yassour, M., Levin, J. Z., Thompson, D. A., Amit, I., et al. (2011). Full-length transcriptome assembly from RNA-seq data without a reference genome. Nat. Biotechnol. 29, 644-652. doi: 10.1038/nbt.1883

Greenway, A. R., and Griffiths, D. C. (1973). A comparison of triglycerides from aphids and their cornicle secretions. J. Insect Physiol. 19, 1649-1655. doi: 10.1016/0022-1910(73)90095-4

Gu, S. H., Wu, K. M., Guo, Y. Y., Pickett, J. A., Field, L. M., Zhou, J. J., et al. (2013). Identification of genes expressed in the sex pheromone gland of the black cutworm Agrotis ipsilon with putative roles in sex pheromone biosynthesis and transport. BMC Genomics 14:636. doi: 10.1186/1471-2164-14-636

Ha, T. S., and Smith, D. P. (2006). A pheromone receptor mediates 11-cisvaccenyl acetate-induced responses in Drosophila. J. Neurosci. 26, 8727-8733. doi: 10.1523/JNEUROSCI.0876-06.2006

Hogenhout, S. A., Oshima, K., Ammar, E. D., Kakizawa, S., Kingdom, H. N., and Namba, S. (2008). Phytoplasmas: bacteria that manipulate plants and insects. Mol. Plant Pathol. 9, 403-423. doi: 10.1111/j.1364-3703.2008.00472.x

Iovinella, I., Dani, F. R., Niccolini, A., Sagona, S., Michelucci, E., Gazzano, A., et al. (2011). Differential expression of odorant-binding proteins in the mandibular glands of the honey bee according to caste and age. J. Proteome Res. 10, 3439-3449. doi: 10.1021/pr2000754

Ishida, Y., Ishibashi, J., and Leal, W. S. (2013). Fatty acid solubilizer from the oral disk of the blowfly. PLoS One 8:e51779. doi: 10.1371/journal.pone.0051779

Jacquin-Joly, E., Vogt, R. G., Francois, M. C., and Nagnan-Le Meillour, P. (2001). Functional and expression pattern analysis of chemosensory proteins expressed in antennae and pheromonal gland of Mamestra brassicae. Chem. Senses 26, 833-844. doi: 10.1093/chemse/26.7.833

Jeong, Y. T., Shim, J., Oh, S. R., Yoon, H. I., Kim, C. H., Moon, S. J., et al. (2013). An odorant-binding protein required for suppression of sweet taste by bitter chemicals. Neuron 79, 725-737. doi: 10.1016/j.neuron.2013.06.025

Johnson, G., Nour, A. A., Nolan, T., Huggett, J., and Bustin, S. (2014). Minimum information necessary for quantitative real-time PCR experiments. Methods Mol. Biol. 1160, 5-17. doi: 10.1007/978-1-4939-0733-5_2

Joshi, N. A., and Fass, J. N. (2011). Sickle: A Sliding-Window, Adaptive, QualityBased Trimming Tool for FastQ Files (Version 1.33) [Software]. Available at https://github.com/najoshi/sickle

Katoh, K., and Standley, D. M. (2013). MAFFT multiple sequence alignment software Version 7: improvements in performance and usability. Mol. Biol. Evol. 30, 772-780. doi: 10.1093/molbev/mst010

Kitabayashi, A. N., Arai, T., Kubo, T., and Natori, S. (1998). Molecular cloning of cDNA for p10, a novel protein that increases in the regenerating legs of Periplaneta americana American cockroach. Insect Biochem. Mol. Biol. 28, 785-790. doi: 10.1016/S0965-1748(98)00058-7

Kruse, S. W., Zhao, R., Smith, D. P., and Jones, D. N. (2003). Structure of a specific alcohol-binding site defined by the odorant binding protein LUSH from Drosophila melanogaster. Nat. Struct. Biol. 10, 694-700. doi: 10.1038/ nsb960

Larter, N. K., Sun, J. S., and Carlson, J. R. (2016). Organization and function of Drosophila odorant binding proteins. eLife 5:e20242. doi: 10.7554/eLife. 20242

Laughlin, J. D., Ha, T. S., Jones, D. N. M., and Smith, D. P. (2008). Activation of pheromone-sensitive neurons is mediate by conformational activation of Pheromone-binding protein. Cell 133, 1255-1265. doi: 10.1016/j.cell.2008. 04.046

Leal, W. S. (2013). Odorant reception in insects: roles of receptors, binding proteins, and degrading enzymes. Annu. Rev. Entomol. 58, 373-391. doi: 10.1146/annurev-ento-120811-153635

Lee, C., Kim, J., Shin, S. G., and Hwang, S. (2006). Absolute and relative QPCR quantification of plasmid copy number in Escherichia coli. J. Biotechnol. 123, 273-280. doi: 10.1016/j.jbiotec.2005.11.014

Li, S., Picimbon, J. F., Ji, S. D., Kan, Y. C., Qiao, C. L., Zhou, J. J., et al. (2008). Multiple functions of an odorant binding protein in the mosquito Aedes aegypti. Biochem. Biophys. Res. Commun. 372, 464-468. doi: 10.1016/j.bbrc.2008.05.064 
Li, Z., Ni, J. D., Huang, J., and Montell, C. (2014). Requirement for Drosophila SNMP1 for rapid activation and termination of pheromoneinduced activity. PLoS Genet. 10:e1004600. doi: 10.1371/journal.pgen.100 4600

Lilley, R., and Hardie, J. (1996). Cereal aphid responses to sex pheromones and host-plant odours in the laboratory. Physiol. Entomol. 21, 304-308. doi: 10.1111/ j.1365-3032.1996.tb00869.x

Liu, W., and Saint, D. A. (2002). A new quantitative method of real time reverse transcription polymerase chain reaction assay based on simulation of polymerase chain reaction kinetics. Anal. Biochem. 302, 52-59. doi: 10.1006/ abio. 2001.5530

Marsh, D. (1972). Sex pheromone in the aphid Megoura viciae. Nat. New Biol. Lond. 238, 31-32. doi: 10.1038/newbio238031a0

Marsh, D. (1975). Responses of male aphids to the female sex pheromone in Megoura viciae Buckton. J. Entomol. Ser. A 50, 43-64. doi: 10.1111/j.1365-3032. 1975.tb00092.x

Martin, M. (2011). Cutadapt removes adapter sequences from high-throughput sequencing reads. EMBnet J. 17, 10-12. doi: 10.14806/ej.17.1.200

Missbach, C., Dweck, H. K., Vogel, H., Vilcinskas, A., Stensmyr, M. C., Hansson, B. S., et al. (2014). Evolution of insect olfactory receptors. eLife 3:e02115. doi: $10.7554 /$ eLife. 02115

Nagnan-Le Meillour, P., Cain, A. H., Jacquin-Joly, E., Francois, M. C., Ramachandran, S., Maida, R., et al. (2000). Chemosensory proteins from the proboscis of Mamestra brassicae. Chem. Senses 25, 541-553. doi: 10.1093/ chemse/25.5.541

Nault, L. R. (1997). Arthropod transmission of plant viruses: a new synthesis. Ann. Entomol. Soc. Am. 90, 521-541. doi: 10.1093/aesa/90.5.521

Nomura, A., Kawasaki, K., Kubo, T., and Natori, S. (1992). Purification and localization of $\mathrm{p} 10$, a novel protein that increases in nymphal regenerating legs of Periplaneta americana American cockroach. Int. J. Dev. Biol. 36, 391-398.

Northey, T., Venthur, H., De Biasio, F., Chauviac, F., Cole, A., and Ribeiro, K. A. Jr. (2016). Crystal structures and binding dynamics of odorant-binding protein 3 from two aphid species Megoura viciae and Nasonovia ribisnigri. Sci. Rep. 6:24739. doi: 10.1038/srep24739

Nuessly, G. S., Hentz, M. G., Beiriger, R., and Scully, B. T. (2004). Insects associated with faba bean, Vicia faba (Fabales:Fabaceae), in southern Florida. Florida Entomol. 87, 204-211. doi: 10.1653/0015-4040(2004)087[0204:IAWFBV]2.0. $\mathrm{CO} ; 2$

Palma, R., Mutis, A., Isaacs, R., and Quiroz, A. (2013). Type and distribution of sensilla in the antennae of the red clover root borer, Hylastinus obscurus. J. Insect Sci. 13:133. doi: 10.1673/031.013.13301

Park, K. C., and Hardie, J. (2004). Electrophysiological characterisation of olfactory sensilla in the black bean aphid, Aphis fabae. J. Insect Physiol. 50, 647-655. doi: 10.1016/j.jinsphys.2004.04.011

Pelletier, J., Guidolin, A., Syed, Z., Cornel, A. J., and Leal, W. S. (2010). Knockdown of a mosquito odorant-binding protein involved in the sensitive detection of oviposition attractants. J. Chem. Ecol. 36, 245-248. doi: 10.1007/s10886-0109762-x

Pelosi, P. (1994). Odorant-binding proteins. Crit. Rev. Biochem. Mol. Biol. 29, 199-228. doi: 10.3109/10409239409086801

Pelosi, P. (1996). Perireceptors events in olfaction. J. Neurobiol. 30, 3-19. doi: 10.1002/(SICI) 1097-4695(199605)30:1<3::AID-NEU2>3.0.CO;2-A

Pelosi, P., Iovinella, I., Zhu, J., Wang, G., and Dani, F. R. (2017). Beyond chemoreception: diverse tasks of soluble olfactory proteins in insects. Biol. Rev. 93, 184-200. doi: 10.1111/brv.12339

Pelosi, P., Zhou, J. J., Ban, L. P., and Calvello, M. (2006). Soluble proteins in insect chemical communication. Cell. Mol. Life Sci. 63, 1658-1676. doi: 10.1007/ s00018-005-5607-0

Pettersson, J. (1971). An aphid sex attractant. II. Histological, ethological and comparative studies. Entomol. Scand. 2, 81-93. doi: 10.1163/187631271X00095

Pickett, J. A., and Griffiths, D. C. (1980). Composition of aphid alarm pheromones. J. Chem. Ecol. 6, 349-360. doi: 10.1007/BF01402913

Pickett, J. A., Wadhams, L. J., Woodcock, C. M., and Hardie, J. (1992). The chemical ecology of aphids. Annu. Rev. Entomol. 37, 67-90. doi: 10.1146/annurev.en.37. 010192.000435

Powell, G., and Hardie, J. (2001). The chemical ecology of aphid host alternation: how do return migrants find the primary host plant? Appl. Entomol. Zool. 36, 259-267. doi: 10.1303/aez.2001.259
Qiao, H. L., Tuccori, E., He, X. L., Gazzano, A., Field, L. M., Zhou, J. J., et al. (2009). Discrimination of alarm pheromone (E)- $\beta$-farnesene by aphid odorant-binding proteins. Insect Biochem. Mol. Biol. 39, 414-419. doi: 10.1016/j.ibmb.2009. 03.004

Quiroz, A., and Niemeyer, H. M. (1998). Olfactometer-assessed responses of aphid Rhopalosiphum padi to wheat and oat volatiles. J. Chem. Ecol. 24, 113-124. doi: 10.1016/j.virusres.2011.04.020

Roitberg, B. D., and Meyers, J. H. (1978). Adaptation of alarm pheromone responses of the pea aphid Acyrthosiphon pisum (Harris). Can. J. Zool. 56, 103-108. doi: 10.1139/z78-014

Schindelin, J., Arganda-Carreras, I., Frise, E., Kaynig, V., Longair, M., Pietzsch, T. et al. (2012). Fiji: an open-source platform for biological-image analysis. Nat. Methods 9, 676-682. doi: 10.1038/nmeth.2019

Shambaugh, G. F., Frazier, J. L., Castell, A. E. M., and Coons, L. B. (1978). Antennal sensilla of seventeen aphid species (Homoptera: Aphidinae). Int. J. Insect Morphol. Embryol. 7, 389-404. doi: 10.1016/S0020-7322(78)80001-4

Shanbhag, S. R., Müller, B., and Steinbrecht, R. A. (2000). Atlas of olfactory organs of Drosophila melanogaster: 2. Internal organization and cellular architecture of olfactory sensilla. Arth. Struct. Dev. 29, 211-229. doi: 10.1016/S1467-8039(00) 00028-1

Sirot, L. K., Poulson, R. L., McKenna, M. C., Girnary, H., Wolfner, M. F., and Harrington, L. C. (2008). Identity and transfer of male reproductive gland proteins of the dengue vector mosquito, Aedes aegypti: potential tools for control of female feeding and reproduction. Insect Biochem. Mol. Biol. 38, 176-189. doi: 10.1016/j.ibmb.2007.10.007

Smartt, C. T., and Erickson, J. S. (2009). Expression of a novel member of the odorant-binding protein gene family in Culex nigripalpus (Diptera: Culicidae). J. Med. Entomol. 46, 1376-1381. doi: 10.1603/033.046.0617

Smith, L. M. (1936). Biology of the mealy plum aphid, Hyalopterus pruni (Geoffroy). Hilgardia 10, 167-211. doi: 10.3733/hilg.v10n07p167

Steinbrecht, R. A. (1997). Pore structures in insect olfactory sensilla: a review of data and concepts. Int. J. Insect Morphol. Embriol. 26, 229-245. doi: 10.1016/ S0020-7322(97)00024-X

Strandh, M., Johansson, T., and Lofstedt, C. (2009). Global transcriptional analysis of pheromone biosynthesis-related genes in the female turnip moth, Agrotis segetum (Noctuidae) using a custom-made cDNA microarray. Insect Biochem. Mol. Biol. 39, 484-489. doi: 10.1016/j.ibmb.2009.04.002

Strong, F. E. (1967). Observations on aphid cornicle secretions. Ann. Entomol. Soc. Am. 60, 668-673. doi: 10.1673/031.010.12601

Stroyan, H. L. G. (1958). A contribution to the taxonomy of some British species of Sappaphis Matsumura 1918 (Homoptera: Aphididae). J. Linn. Soc. 43, 644-713. doi: $10.1111 / j .1096-3642.1958 . t b 01582 . x$

Sun, Y. F., De Biasio, F., Qiao, H. L., Iovinella, I., Yang, S. X., Ling, Y., et al. (2012). Two odorant-binding proteins mediate the behavioral response of aphids to the alarm pheromone (E)- $\beta$-farnesene and structural analogues. PLoS One 7:e32759. doi: 10.1371/journal.pone.0032759

Sun, Y. F., Qiao, H. L., Ling, Y., Yang, S., Rui, C., Pelosi, P., et al. (2011). New analogues of (E)- $\beta$-farnesene with insecticidal activity and binding affinity to aphid odorant-binding proteins. Agric. Food Chem. 59, 2456-2461. doi: $10.1021 /$ jf $104712 \mathrm{c}$

Sun, Y. L., Huang, L. Q., Pelosi, P., and Wang, C. Z. (2012). Expression in antennae and reproductive organs suggests a dual role of an odorant-binding protein in two sibling Helicoverpa species. PLoS One 7:e30040. doi: 10.1371/journal.pone. 0030040

Sun, Y. P., Zhao, L. J., Sun, L., Zhang, S. G., and Ban, L. P. (2013). Immunolocalization of odorant-binding proteins on antennal chemosensilla of the peach aphid Myzus persicae (Sulzer). Chem. Senses 38, 129-136. doi: 10.1093/chemse/bjs093

Swarup, S., Morozova, T. V., Sridhar, S., Nokes, M., and Anholt, R. R. H. (2014). Modulation of feeding behavior by odorant-binding proteins in Drosophila melanogaster. Chem. Senses 39, 125-132. doi: 10.1093/chemse/bjt061

Swarup, S., Williams, T. I., and Anholt, R. R. (2011). Functional dissection of odorant binding protein genes in Drosophila melanogaster. Genes Brain Behav. 10, 648-657. doi: 10.1111/j.1601-183X.2011.00704.x

van Emden, H. F., and Harrington, R. (2007). Aphids as Crop Pests. Wallingford: CAB International. doi: 10.1079/9780851998190.0000

van Giessen, W. A., Fescemyer, H. W., Burrows, P. M., Peterson, J. K., and Barnett, O. W. (1994). Quantification of electroantennogram responses of the 
primary rhinaria of Acyrthosiphon pisum (Harris) to C4-C8 primary alcohols and aldehydes. J. Chem. Ecol. 20, 909-927. doi: 10.1007/BF02059587

Vandermoten, S., Francis, F., Haubruge, E., and Leal, W. S. (2011). Conserved odorant-binding proteins from aphids and eavesdropping predators. PLoS One 6:e23608. doi: 10.1371/journal.pone.0023608

Vogel, H., Heidel, A. J., Heckel, D. G., and Groot, A. T. (2010). Transcriptome analysis of the sex pheromone gland of the noctuid moth Heliothis virescens. BMC Genomics 11:29. doi: 10.1186/1471-2164-11-29

Vogt, R. G., Prestwich, G. D., and Lerner, M. R. (1991). Odorant-binding-protein subfamilies associate with distinct classes of olfactory receptor neurons in insects. J. Neurobiol. 22, 74-84. doi: 10.1002/neu.480220108

Vogt, R. G., and Riddiford, L. M. (1981). Pheromone binding and inactivation by moth antennae. Nature 293, 161-163. doi: 10.1038/293161a0

Weber, H. (1935). In Hemiptera. III. Biologie der Tiere Deutschlands 38, 210-286.

Webster, B. (2012). The role of olfaction in aphid host location. Physiol. Entomol. 37, 10-18. doi: 10.1111/j.1365-3032.2011.00791.x

Wohlers, P., and Tjallingii, W. F. (1983). Electroantennogram responses of aphids to the alarm pheromone E- $\beta$-farnesene. Entomol. Exp. Appl. 331, 79-82. doi: 10.1111/j.1570-7458.1983.tb03236.x

Xia, Y. H., Zhang, Y. N., Hou, X. Q., Li, F., and Dong, S. L. (2015). Large number of putative chemoreception and pheromone biosynthesis genes revealed by analyzing transcriptome from ovipositor-pheromone glands of Chilo suppressalis. Sci. Rep. 5:7888. doi: 10.1038/srep07888

Xu, P., Atkinson, R., Jones, D. N., and Smith, D. P. (2005). Drosophila OBP LUSH is required for activity of pheromone-sensitive neurons. Neuron 45, 193-200. doi: 10.1016/j.neuron.2004.12.031

Zhang, R., Wang, B., Grossi, G., Falabella, P., Liu, Y., Yan, S., et al. (2017). Molecular basis of alarm pheromone detection in aphids. Curr. Biol. 27, 55-61. doi: 10.1016/j.cub.2016.10.013

Zhang, Y. N., Jin, J. Y., Jin, R., Xia, Y. H., Zhou, J. J., Deng, J. Y., et al. (2013). Differential expression patterns in chemosensory and non-chemosensory tissues of putative chemosensory genes identified by transcriptome analysis of insect pest the purple stem borer Sesamia inferens (Walker). PLoS One 8:e69715. doi: 10.1371/journal.pone.0069715

Zhang, Y. N., Zhu, X. Y., Fang, L. P., He, P., Wang, Z. Q., Chen, G., et al. (2015). Identification and expression profiles of sex pheromone biosynthesis and transport related genes in Spodoptera litura. PLoS One 10:e0140019. doi: 10.1371/journal.pone.0140019

Zhou, J. J., Huang, W., Zhang, G. A., Pickett, J. A., and Field, L. M. (2004). "PlusC" odorant-binding protein genes in two Drosophila species and the malaria mosquito Anopheles gambiae. Gene 327, 117-129. doi: 10.1016/j.gene.2003. 11.007

Zhou, J. J., Vieira, F. G., He, X. L., Smadja, C., Liu, R., Rozas, J., et al. (2010). Genome annotation and comparative analyses of the odorant-binding proteins and chemosensory proteins in the pea aphid Acyrthosiphon pisum. Insect Mol. Biol. 19, 113-122. doi: 10.1111/j.1365-2583.2009. 00919.x

Ziegelberger, G. (1995). Redox-shift of the pheromone-binding protein in the silkmoth Antheraea polyphemus. Eur. J. Biochem. 232, 706-711. doi: 10.1111/ j.1432-1033.1995.tb20864.x

Conflict of Interest Statement: The authors declare that the research was conducted in the absence of any commercial or financial relationships that could be construed as a potential conflict of interest.

Copyright (c) 2018 Bruno, Grossi, Salvia, Scala, Farina, Grimaldi, Zhou, Bufo, Vogel, Grosse-Wilde, Hansson and Falabella. This is an open-access article distributed under the terms of the Creative Commons Attribution License (CC BY). The use, distribution or reproduction in other forums is permitted, provided the original author(s) and the copyright owner are credited and that the original publication in this journal is cited, in accordance with accepted academic practice. No use, distribution or reproduction is permitted which does not comply with these terms. 